This item was submitted to Loughborough's Research Repository by the author.

Items in Figshare are protected by copyright, with all rights reserved, unless otherwise indicated.

\title{
Electrified viscous thin film flow over topography
}

PLEASE CITE THE PUBLISHED VERSION

http://dx.doi.org/10.1017/S002211200700986X

PUBLISHER

(c) Cambridge University Press

VERSION

VoR (Version of Record)

LICENCE

CC BY-NC-ND 4.0

REPOSITORY RECORD

Tseluiko, Dmitri, Mark G. Blyth, Demetrios T. Papageorgiou, and Jean-Marc Vanden-Broeck. 2019. "Electrified Viscous Thin Film Flow over Topography". figshare. https://hdl.handle.net/2134/15489. 
This item was submitted to Loughborough's Institutional Repository (https://dspace.lboro.ac.uk/) by the author and is made available under the following Creative Commons Licence conditions.

\section{creative
commons}

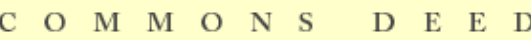

Attribution-NonCommercial-NoDerivs 2.5

You are free:

- to copy, distribute, display, and perform the work

Under the following conditions:

Attribution. You must attribute the work in the manner specified b the author or licensor.

Noncommercial. You may not use this work for commercial purposes.

No Derivative Works. You may not alter, transform, or build upon this work.

- For any reuse or distribution, you must make clear to others the license terms of this work.

- Any of these conditions can be waived if you get permission from the copyright holder.

Your fair use and other rights are in no way affected by the above.

This is a human-readable summary of the Leqal Code (the full license).

\section{Disclaimer 만}

For the full text of this licence, please go to: http://creativecommons.org/licenses/by-nc-nd/2.5/ 


\title{
Electrified viscous thin film flow over topography
}

\author{
D. TSELUIK O ${ }^{1}$, M. G. BL Y T H ${ }^{1}$, \\ D. T. PAPA GEOR GIOU ${ }^{2}$ AND J. - M. VANDEN-BROE CK \\ ${ }^{1}$ School of Mathematics, University of East Anglia, Norwich, NR4 7TJ, UK \\ ${ }^{2}$ Department of Mathematical Sciences and Center for Applied Mathematics and Statistics, \\ New Jersey Institute of Technology, Newark, NJ 07102, USA
}

(Received 19 April 2007 and in revised form 7 November 2007)

The gravity-driven flow of a liquid film down an inclined wall with periodic indentations in the presence of a normal electric field is investigated. The film is assumed to be a perfect conductor, and the bounding region of air above the film is taken to be a perfect dielectric. In particular, the interaction between the electric field and the topography is examined by predicting the shape of the film surface under steady conditions. A nonlinear, non-local evolution equation for the thickness of the liquid film is derived using a long-wave asymptotic analysis. Steady solutions are computed for flow into a rectangular trench and over a rectangular mound, whose shapes are approximated with smooth functions. The limiting behaviour of the film profile as the steepness of the wall geometry is increased is discussed. Using substantial numerical evidence, it is established that as the topography steepness increases towards rectangular steps, trenches, or mounds, the interfacial slope remains bounded, and the film does not touch the wall. In the absence of an electric field, the film develops a capillary ridge above a downward step and a slight depression in front of an upward step. It is demonstrated how an electric field may be used to completely eliminate the capillary ridge at a downward step. In contrast, imposing an electric field leads to the creation of a free-surface ridge at an upward step. The effect of the electric field on film flow into relatively narrow trenches, over relatively narrow mounds, and down slightly inclined substrates is also considered.

\section{Introduction}

Controlling the flow of a liquid film down a smooth or corrugated plate is of fundamental importance in a broad range of engineering applications, including the manufacture of photographic plates and magnetic memory devices. In industrial coating, a liquid film is continuously deposited onto a moving substrate to produce a thin layer of uniform thickness (e.g. Kistler \& Schweizer 1997). Natural imperfections in the target surface may lead to undesirable ripples or variations in the thickness of the coated layer (e.g. Stillwagon \& Larson 1988, 1990). In some applications, an uneven film surface may be a desirable outcome. For example, in liquid film cooling surface waves can significantly enhance heat or mass transfer (e.g. Yoshimura, Nosoko \& Nagata 1996; Serifi, Malamataris \& Bontozoglou 2004). For these reasons, a great deal of effort has been devoted to the theoretical prediction of film flow behaviour under a wide variety of conditions.

The linear stability of film flow down an inclined plane was analysed by Benjamin (1957) and Yih (1963). They showed that there exists a critical Reynolds number above which the flow becomes unstable to long waves. This result was verified experimentally 
by Liu, Paul \& Gollub (1993) and Liu \& Gollub (1994). Fully computational studies of waves occurring on the surface of a flowing film include those by Ramaswamy, Chippada \& Joo (1996) and Malamataris, Vlachogiannis \& Bontozoglou (2002). The daunting complexity of the full nonlinear problem, even in the absence of topographically structured substrates, led a number of workers to derive reduced models valid for long waves (e.g. Benney 1966; Lin 1974; Alekseenko, Nakoryakov \& Pokusaev 1985). Such models have proved successful in describing experimental observations, including the formation of capillary ridges close to a contact line at the front of a flowing film (e.g. Bertozzi \& Brenner 1997; Diez \& Kondic 2001).

The linear stability of weakly nonlinear long waves on a film flowing along an inclined uneven wall were studied by Tougou (1978). Wang (1981) performed an asymptotic analysis for the flow of a film over a sinusoidal wall when the film thickness is much larger than the wall amplitude. In both of these studies, the amplitude of the wall corrugations was assumed small. This assumption was relaxed by Wang (1984), who considered film flow along a curved surface whose minimum radius of curvature is much larger than the film thickness, and by Shetty \& Cerro (1993), who also compared their results with experiments. Numerous computational and experimental studies have addressed the problem of film flow along a corrugated wall. Pozrikidis (1988) used the boundary-integral method to compute steady film flow at zero Reynolds number over a variety of wall shapes. Trifonov (1999), Malamataris \& Bontozoglou (1999), and Gu et al. (2004) computed falling-film flow over an uneven wall at arbitrary Reynolds number and produced results in good agreement with experimental work, e.g. Zhao \& Cerro (1992). Experimental results for gravity-driven film flow down an inclined wall with periodic rectangular corrugations were reported by Messé \& Decré (1997), Fernandez-Parent, Lammers \& Decré (1998), Decré \& Baret (2003), Vlachogiannis \& Bontozoglou (2002), and Argyriadi, Vlachogiannis \& Bontozoglou (2006). The formation of eddies at very low Reynolds numbers of order $10^{-5}$ for flows over undulating walls was studied experimentally by Wierschem, Scholle \& Aksel (2003). They found that eddies form when the film thickness is above a critical value, which depends on the waviness of the wall, the angle of inclination, and the surface tension, but not on the Reynolds number. Additional experiments of film flow over moderate substrate undulations were carried out by Wierschem \& Aksel (2004a) and Wierschem \& Aksel (2004b); the latter study considers the effect of inertia on eddies formed in the creeping flow regime described in Wierschem et al. (2003).

Derivations of thin-film equations over curved substrates can be found in the work of Schwartz \& Weidner (1995), Roy, Roberts \& Simpson (2002), and Howell (2003). Flow of a liquid film over an abrupt step or over a rectangular trench was investigated by Kalliadasis, Bielarz \& Homsy (2000) and Bielarz \& Kalliadasis (2003) using the lubrication model of Stillwagon \& Larson (1990). Their results showed that the freesurface shape generally follows the topography of the wall but can exhibit significant variations near to an upward or a downward step. A pronounced capillary ridge develops directly above a downward step, and a slight depression forms in front of an upward step. Both of these features were observed experimentally by Messé \& Decré (1997), Fernandez-Parent et al. (1998), and Decré \& Baret (2003) and were captured by the boundary-integral calculations of Mazouchi \& Homsy (2001). The latter work lends credence to the results obtained using the lubrication model despite reservations about its applicability for flow over steep topography. In a more recent related study, Gaskell et al. (2004) (for a study that also includes evaporation, see Gaskell et al. 2006) extended the work of Mazouchi \& Homsy (2001) to non-zero Reynolds 
numbers. They used two-dimensional topography-following lubrication models as well as three-dimensional finite-element simulations of the Navier-Stokes equations to study film flow over smoothed but still relatively sharp rectangular indentations. They found that at steady state an increase in the Reynolds number increases the amplitude of the ridge formed ahead of a downward step and decreases the size of the depression found ahead of an upward step. The predictions of the lubrication models, the Navier-Stokes simulations, and the experimental results of Decre \& Baret (2003) are all found to be in very good agreement, increasing confidence in the use of model equations for these types of flow. Related studies of creeping film flow in a corner or over a hump can be found in Stocker \& Hosoi (2005) and Jensen, Chini \& King (2004), who study thin film flow over topography in the absence of a driving gravitational force, in contrast to the present study and the aforementioned references.

The manipulation of a liquid film by means of an electric field has been discussed by numerous authors with particular applications in mind, for example, by Bankoff et al. (1994), Kim, Bankoff \& Miksis (1994), Bankoff et al. (2002), and Griffing et al. (2006). The electric field affects the flow through an additional Maxwell stress term in the stress balance at the film surface. Film flow down an inclined plane in the presence of a normal electric field was studied by Gonzalez \& Castellanos (1996) and Tseluiko \& Papageorgiou (2006). The film was assumed to be a perfect conductor, and the medium above the film was assumed to be a perfect dielectric. The electric field was viewed as being imposed by an infinite electrode placed a long distance from the film. Using a long-wave theory, they derived evolution equations describing the nonlinear film dynamics and demonstrated the existence of travelling wave solutions, periodic homoclinic bursts, and chaotic oscillations. The general conclusion is that the effect of the electric field is destabilizing. The effect of imposing the electric field via a finite-length electrode was considered by Kim, Bankoff \& Miksis (1992). Recently, Papageorgiou \& Petropoulos (2004) used a leaky dielectric model to study the stability of an electrified film at finite Reynolds number.

In the present work, we examine steady film flow over an indented wall when the film is subjected to a normal electric field. The film is assumed to be a perfect conductor, and the air above the film is assumed to be a perfect dielectric. Our particular goal is to assess the effect of the electric field on the shape of the free surface. As discussed above, either a smooth or a rippled film may be required in practical applications. We investigate when an electric field is able to eliminate unwanted features, such as the capillary ridge, and when it tends instead to promote the deformation of the film surface. Motivated by the success of the lubrication model in describing the salient features of film flow in the absence of an electric field, we use a long-wave theory to derive a nonlinear evolution equation for the film height. We focus our attention on steady solutions to this equation in the case of flow over upward and downward steps, and flow into rectangular trenches and over rectangular mounds.

In $\S 2$, we describe the physical problem and present the governing equations. In $\S 3$, we use an asymptotic long-wave theory to derive a nonlinear evolution equation for the interface. Section 4 is devoted to studying steady-state solutions of this equation. Finally, in $\S 5$, we present our conclusions.

\section{Problem formulation}

We consider the two-dimensional, gravity-driven flow of a liquid film down an uneven wall, which is inclined at an angle $\beta$ to the horizontal, when the film is 


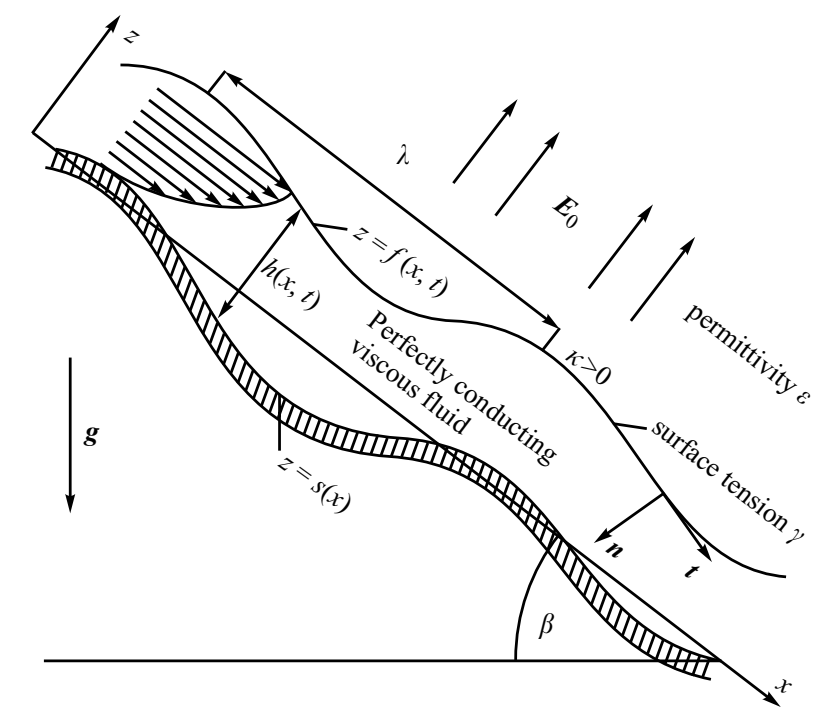

FIGURE 1. Schematic of the problem: flow of a fluid film down a corrugated wall in the presence of gravity and an electric field.

subjected to a normal electric field, as is illustrated in figure 1 . With reference to the Cartesian axes shown in figure 1, the wall topography is described by the equation $z=s(x)$, and the film thickness is given by $h(x, t)$, so that the film surface is located at $z=f(x, t) \equiv s(x)+h(x, t)$. The film is subjected to a uniform normal electric field of strength $E_{0}$ imposed a long way from the wall, as is shown in figure 1 . The air above the film is assumed to be a perfect dielectric with permittivity $\varepsilon$. The electric potential above the film is denoted by $\varphi(x, z, t)$ and satisfies the relation $\boldsymbol{E}=-\nabla \varphi$, where $\boldsymbol{E}$ is the electric field. The liquid is assumed to be a perfect conductor, so that there is no potential difference between the wall and the free surface and, consequently, no electric field in the film.

The governing equations in the film are the Navier-Stokes equation and the continuity equation,

$$
\rho\left(\frac{\partial \boldsymbol{u}}{\partial t}+\boldsymbol{u} \cdot \nabla \boldsymbol{u}\right)=-\nabla p+\mu \nabla^{2} \boldsymbol{u}+\rho \boldsymbol{g}, \quad \nabla \cdot \boldsymbol{u}=0,
$$

where $\boldsymbol{u}=(u, v)$ is the fluid velocity, $p$ is the pressure, $\rho$ and $\mu$ are the density and viscosity of the liquid, respectively, and $\boldsymbol{g}$ is gravity. In the air above the film, the electric potential, $\varphi$, satisfies Laplace's equation,

$$
\nabla^{2} \varphi=0 .
$$

The no-slip and the impermeability boundary conditions require that

$$
u=0, \quad v=0
$$

at the wall, $z=s(x)$. The uniform electric field condition at infinity requires that

$$
\nabla \varphi \rightarrow-E_{0} \boldsymbol{k}
$$

as $z \rightarrow \infty$, where $\boldsymbol{k}$ is the unit vector in the $z$-direction. At the film surface, $z=f(x, t)$, we have the kinematic condition,

$$
v=f_{t}+u f_{x}
$$


the zero potential condition,

$$
\varphi=0
$$

and the dynamic balance of stress,

$$
\boldsymbol{\sigma} \cdot \boldsymbol{n}=-\left(\gamma \kappa+p_{a}\right) \boldsymbol{n}+\boldsymbol{M} \cdot \boldsymbol{n},
$$

where $\sigma$ is the Newtonian stress tensor, $\gamma$ is the surface tension, $p_{a}$ is the atmospheric pressure, and $\boldsymbol{n}$ is the unit normal to the free surface pointing into the film. The freesurface curvature, $\kappa$, is taken to be positive when the surface is concave downwards, as is shown in figure 1 . The Maxwell stress tensor, $\boldsymbol{M}$, is defined by

$$
\boldsymbol{M}=\varepsilon\left(\boldsymbol{E} \boldsymbol{E}-\frac{1}{2} \boldsymbol{I}|\boldsymbol{E}|^{2}\right)
$$

It may be verified directly that the Maxwell stresses do not contribute to the tangential stress balance. In fact, the normal and tangential stress balance conditions at the free surface are

$$
\boldsymbol{t} \cdot \boldsymbol{\sigma} \cdot \boldsymbol{n}=0, \quad \boldsymbol{n} \cdot \boldsymbol{\sigma} \cdot \boldsymbol{n}=-\left(\gamma \kappa+p_{a}\right)+\frac{\varepsilon}{2}|\boldsymbol{E}|^{2},
$$

where $t$ is the unit tangent to the free surface, depicted in figure 1. It will be noted that the Maxwell stress term in $(2.9 b)$ is non-negative and, hence, effectively decreases the local ambient pressure felt by any point on the film surface.

To simplify the presentation, we introduce the shifted pressure, $\tilde{p}$, and electric potential, $\tilde{\varphi}$, by writing

$$
\begin{aligned}
p & =\tilde{p}+p_{\mathrm{a}}-\frac{\varepsilon E_{0}^{2}}{2}+\rho \mathrm{g} x \sin \beta-\rho \mathrm{g} z \cos \beta, \\
\varphi & =\tilde{\varphi}+E_{0} h_{0}-E_{0} z,
\end{aligned}
$$

where $h_{0}$ is the typical film thickness. These definitions remove the gravity terms from (2.1a) and simplify the normal stress balance (2.9b). The shifted potential, $\tilde{\varphi}$, describes the deviation from a wholly uniform electric field. Proceeding, we non-dimensionalize variables by writing

$$
\left.\begin{array}{c}
x^{*}=\frac{1}{h_{0}} x, \quad z^{*}=\frac{1}{h_{0}} z, \quad t^{*}=\frac{U_{0}}{h_{0}} t, \quad f^{*}=\frac{1}{h_{0}} f, \quad s^{*}=\frac{1}{h_{0}} s, \\
u^{*}=\frac{1}{U_{0}} u, \quad v^{*}=\frac{1}{U_{0}} v, \quad p^{*}=\frac{h_{0}}{\mu U_{0}} \tilde{p}, \quad \varphi^{*}=\frac{1}{E_{0} h_{0}} \tilde{\varphi},
\end{array}\right\}
$$

where an asterisk denotes a dimensionless quantity and $U_{0}=\rho \mathrm{g} h_{0}^{2} \sin \beta / 2 \mu$ is the Nusselt surface speed of a flat film flowing down an inclined plane. In addition, we introduce the Reynolds number, $R$, the capillary number, $C$, and the electric Weber number, $W_{e}$, measuring the relative importance of electric forces to viscous forces,

$$
\begin{gathered}
R=\frac{\rho U_{0} h_{0}}{\mu}=\frac{\rho^{2} \mathrm{~g} h_{0}^{3} \sin \beta}{2 \mu^{2}}, \quad C=\frac{\mu U_{0}}{\gamma}=\frac{\rho \mathrm{g} h_{0}^{2} \sin \beta}{2 \gamma}, \\
W_{e}=\frac{\varepsilon h_{0} E_{0}^{2}}{2 \mu U_{0}}=\frac{\varepsilon E_{0}^{2}}{\rho \mathrm{g} h_{0} \sin \beta} .
\end{gathered}
$$

Substituting (2.10), (2.11), and (2.12) into (2.1a,b) and (2.2), and also into (2.3a,b)(2.6) and $(2.9 a, b)$, and dropping the asterisks, we obtain

$$
\begin{aligned}
R\left(u_{t}+u u_{x}+v u_{z}\right) & =-p_{x}+u_{x x}+u_{z z}, \\
R\left(v_{t}+u v_{x}+v v_{z}\right) & =-p_{z}+v_{x x}+v_{z z}, \\
u_{x}+v_{z} & =0,
\end{aligned}
$$


in the liquid film, and

$$
\varphi_{x x}+\varphi_{z z}=0
$$

above the film. The conditions at the wall are given by $u=0$ and $v=0$ at $z=s(x)$, and at infinity we have

$$
\varphi_{x} \rightarrow 0, \quad \varphi_{z} \rightarrow 0 \quad \text { as } \quad z \rightarrow \infty .
$$

At the film surface, $z=f(x, t)$, the kinematic condition becomes

$$
v=f_{t}+u f_{x},
$$

the zero potential condition becomes

$$
\varphi=f-1,
$$

the tangential stress balance is given by

$$
\left(1-f_{x}^{2}\right)\left(u_{z}+v_{x}\right)+4 f_{x} v_{z}=0,
$$

and the normal stress balance is given by

$$
-\frac{W_{e}}{2}\left[\left(1-\varphi_{z}\right)^{2}\left(1+f_{x}^{2}\right)-1\right]+\frac{1+f_{x}^{2}}{1-f_{x}^{2}} v_{z}-\frac{1}{2}(p+2 x-2 f \cot \beta)=\frac{f_{x x}}{2 C\left(1+f_{x}^{2}\right)^{3 / 2}} .
$$

It will be noted that we have made use of (2.21) in deriving (2.22).

\section{Thin-film analysis}

We now assume that the film flow over the corrugated wall is such that streamwise variations occur over a much longer length scale, $\lambda$, than the typical film thickness, $h_{0}$, and work on the basis of the lubrication approximation. Our goal is to derive a nonlinear evolution equation for the free surface. Introducing the small parameter $\delta=h_{0} / \lambda$, we rescale the electric problem by writing

$$
x=\frac{1}{\delta} \xi, \quad z=\frac{1}{\delta} \eta .
$$

The perturbation voltage potential satisfies

$$
\varphi_{\xi \xi}+\varphi_{\eta \eta}=0,
$$

with $\varphi_{\xi} \rightarrow 0$ and $\varphi_{\eta} \rightarrow 0$ as $\eta \rightarrow \infty$, and with $\varphi=f-1$ on $\eta=0$. The last condition is an approximation based on the assumption that $\delta$ is small. Using complex variable theory it can be shown that

$$
\varphi_{\eta}(\xi, 0)=-\mathscr{H}\left[f_{\xi}\right]
$$

(see Papageorgiou \& Vanden-Broeck 2004; Tseluiko \& Papageorgiou 2006), where $\mathscr{H}$ is the Hilbert transform operator defined as

$$
\mathscr{H}[g](\xi)=\frac{1}{\pi} P V \int_{-\infty}^{\infty} \frac{g\left(\xi^{\prime}\right)}{\xi-\xi^{\prime}} \mathrm{d} \xi^{\prime},
$$

and $P V$ denotes the principal value of the integral. The pertinent properties of the Hilbert transform required for the present work may be found in Abdelouhab et al. (1989), for example. Correct to leading order, we take

$$
\varphi_{z}=-\delta \mathscr{H}\left[f_{\xi}\right]
$$

in the normal stress balance condition (2.22). 
In the liquid film, we change variables by writing

$$
x=\frac{1}{\delta} \xi, \quad z=z, \quad t=\frac{1}{\delta} \tau, \quad v=\delta w
$$

and expand the flow variables as follows:

$$
\begin{array}{ll}
u=U+O(\delta), & w=W+O(\delta), \\
p=\delta^{-1} P+O(1), & f=F+O(\delta) .
\end{array}
$$

To retain both the electric field and surface tension effects, we assume that

$$
C=\delta^{3} C^{\prime}, \quad W_{e}=\frac{W_{e}^{\prime}}{\delta^{2}},
$$

where $C^{\prime}=O(1)$ and $W_{e}^{\prime}=O(1)$. Neglecting the gravitational component in the $z$ direction corresponds to assuming that $\cot \beta \ll \delta^{-1}$. We make the further assumption that $R \ll \delta^{-1}$, which is inclusive of the case of Stokes flow. It will be noted that these assumptions differ from those adopted by Tseluiko \& Papageorgiou (2006). Moreover, Tseluiko \& Papageorgiou (2006) also retained the $z$-component of gravity in their thin-film analysis and assumed that the Reynolds number is of order one.

Proceeding, we substitute the expansions (3.7) into (2.14)-(2.16), the zero-velocity conditions at the wall, the kinematic condition (2.19), and the tangential and normal stress balance conditions (2.21) and (2.22), respectively, and derive the following system at leading order,

$$
U_{z z}=P_{\xi}, \quad P_{z}=0, \quad W_{z}=-U_{\xi},
$$

with $U=0$ and $W=0$ on the wall, $z=s(\xi)$, and with

$$
\begin{aligned}
& F_{\tau}=W-U F_{\xi}, \\
& U_{z}=0, \\
& P=-2 \xi-\frac{1}{C^{\prime}} F_{\xi \xi}-2 W_{e}^{\prime} \mathscr{H}\left[F_{\xi}\right]
\end{aligned}
$$

at the film surface, $z=F(\xi, \tau)$. The solution is

$$
\begin{aligned}
U & =\frac{P_{\xi}}{2}(z-s)^{2}-P_{\xi}(F-s)(z-s), \\
W & =-\frac{P_{\xi \xi}}{6}(z-s)^{3}+\frac{\left(P_{\xi}(F-s)\right)_{\xi}+P_{\xi} s_{\xi}}{2}(z-s)^{2}-P_{\xi} s_{\xi}(F-s)(z-s),
\end{aligned}
$$

where $P$ is given by (3.12) throughout the film. Substituting (3.13) and (3.14) into the kinematic condition (3.10) and writing $H=F-s$ yields the evolution equation for the leading-order film thickness

$$
H_{\tau}=\frac{1}{3}\left[H^{3} P_{\xi}\right]_{\xi},
$$

which can alternatively be expressed as

$$
H_{\tau}+\left[\frac{2}{3} H^{3}+\frac{1}{3 C^{\prime}} H^{3}(H+s)_{\xi \xi \xi}+\frac{2}{3} W_{e}^{\prime} H^{3} \mathscr{H}\left[(H+s)_{\xi \xi}\right]\right]_{\xi}=0 .
$$

Stillwagon \& Larson (1990) derived a similar equation, but without the electric field term, for the case of axisymmetric flow of a thin fluid layer over a rotating disk with axisymmetric corrugations. The analogous equation (containing a sign error), 
describing the surface-tension-driven flow of a viscous liquid film over a substrate at zero Reynolds number and in the absence of an electric field, was also obtained by Stillwagon \& Larson (1988). Thin-film equations involving a Hilbert transform term have also arisen in crack propagation studies (e.g. Spence, Sharp \& Turcotte 1987; Lister 1990).

\section{Steady-state solutions}

In this section, we compute free-surface profiles for steady electrified film flow over topography. Previous workers have considered this problem in the absence of an electric field. Kalliadasis et al. (2000) applied the lubrication model of Stillwagon \& Larson (1990) to compute steady film flow into a trench and found that the free surface exhibits a well-defined ridge just upstream of the downward step. The presence of the ridge was confirmed by Mazouchi \& Homsy (2001) for Stokes flow using a boundary-integral method. In fact, most of the important flow features, including the formation of the capillary ridge just described, the thinning of the film close to a downward or an upward step, and the occurrence of a depression immediately in front of an upward step, are captured without difficulty by the lubrication theory, despite its theoretical limitations. We do not, however, expect the lubrication model to accurately reproduce the flow within the film. For example, the flow over a rectangular trench to be considered in $\S 4.3$ should in reality feature viscous eddies inside the film. According to (3.12) and (3.13), $U$ has the same sign for all $z$ at any given downstream location and so the possibility of eddies is excluded in the current model.

Motivated by the previous success of the lubrication model in predicting freesurface profiles, we use a numerical method to study solutions to the steady form of the evolution equation (3.16),

$$
\left[\frac{2}{3} H^{3}+\frac{1}{3 C^{\prime}} H^{3}(H+s)_{\xi \xi \xi}+\frac{2}{3} W_{e}^{\prime} H^{3} \mathscr{H}\left[(H+s)_{\xi \xi}\right]\right]_{\xi}=0,
$$

for flow over several different classes of topography, including a rectangular trench, a single downward or upward step, and a rectangular mound. We compute solutions on the finite interval $[-L, L]$, for a chosen value of $L$, assuming periodic boundary conditions at the ends. Under the assumed $2 L$-periodicity of the solution, the Hilbert transform (3.4) adopts the particular form

$$
\mathscr{H}[f](\xi)=\frac{1}{2 L} P V \int_{-L}^{L} f\left(\xi^{\prime}\right) \cot \left(\frac{\pi\left(\xi-\xi^{\prime}\right)}{2 L}\right) \mathrm{d} \xi^{\prime} .
$$

The finite interval is divided into a uniform grid with $2 M$ mesh points defined by

$$
\xi_{i}=(i-M) \Delta \xi
$$

for $i=1,2, \ldots, 2 M$, where $\Delta \xi=L / M$ is the grid spacing. The value of $H$ at the $i$ th mesh point is denoted by $H_{i}$. The spatial derivatives in (4.1) are approximated using second-order-accurate central differences. The periodicity demands that $H_{0}=H_{2 M}$, $H_{2 M+1}=H_{1}$, and so on. The Hilbert transform of $(H+s)_{\xi \xi}$ at the midpoints $\xi_{i+1 / 2}=\left(\xi_{i}+\xi_{i+1}\right) / 2$, for $i=0,1, \ldots, 2 M-1$, is calculated by using the trapezoidal rule with the $\xi_{i}$ as integration nodes. In this way, the principal value integral in (4.2) is efficiently computed, as if it were simply an ordinary integral. Applying the discretized form of (4.1) at each of the mesh points, we obtain a system of $2 \mathrm{M}$ nonlinear algebraic equations for the $2 M$ unknowns $H_{1}, H_{2}, \ldots, H_{2 M}$. Since (4.1) 
may be integrated once to furnish an arbitrary constant, we are free to fix one of the unknowns in advance. Typically, we fix $H_{2 M}=1$ and remove one of the equations to leave a system of $2 M-1$ equations for the $2 M-1$ unknowns $H_{1}, H_{2}, \ldots, H_{2 M-1}$, which is solved using Newton's method. The value of $M$ is chosen to be sufficiently large to accurately resolve the film profile and the wall shape. For the calculations presented in this paper we took values of $M$ up to 3000 .

To study film flow over upward or downward steps, we approximate the sharp topography using hyperbolic tangent functions, which are suited to the periodic boundary conditions presently considered. Accordingly, a true rectangular trench described by

$$
s_{0}(\xi)=2 D\left[H_{v}(\xi-B)-H_{v}(\xi+B)\right],
$$

where $H_{v}$ is the Heaviside function and $D$ and $B$ are chosen constants, is approximated by the formula

$$
s(\xi)=D\left[\tanh \left(\frac{\xi-B}{d}\right)-\tanh \left(\frac{\xi+B}{d}\right)\right],
$$

for an appropriate choice of the parameter $d$. A true rectangular mound described by

$$
s_{0}(\xi)=-2 D\left[1+H_{v}(\xi-B)-H_{v}(\xi+B)\right]
$$

is modelled by the formula

$$
s(\xi)=-D\left[2+\tanh \left(\frac{\xi-B}{d}\right)-\tanh \left(\frac{\xi+B}{d}\right)\right],
$$

for a suitable choice of $d$. We note that the periodic extension of the wall shape $s(\xi)$ is non-smooth at the ends. However, for computational purposes, since $s( \pm L)=O\left(e^{-2 L / d}\right)$, it follows that for sufficiently large $L$ or small $d$ the wall shape is effectively smooth to within machine precision. Indeed, careful checks have been performed to ensure that the wall shape is effectively periodic to within machine accuracy for all the results presented in this paper. This was done by monitoring the Fourier spectra of $s(\xi), H(\xi)$, and $F(\xi)$ for each discretization, which showed the appropriate exponential decay down to machine round-off error.

Solitary downward and upward steps are recovered in the double limit $L \rightarrow \infty$ and $B \rightarrow \infty$. Sharp ninety-degree steps are obtained in the limit $d \rightarrow 0$. Approximating the latter limit numerically is a matter of some subtlety, as we shall discuss next.

\subsection{Film flow over a sharp corner}

To study the numerical solution for film flow in the presence of a sharp step, we perform calculations for small values of $d$. Throughout this section, we consider film flow over the rectangular trench obtained by fixing $D=0.75$ and $B=4$ in the wall shape formula (4.5) for the case $C^{\prime}=1$ and $W_{e}^{\prime}=1$. As a preliminary, we confirmed that as $L$ increases for a fixed value of $d$, the film surface converges to a limiting profile and the velocity field approaches the expected Nusselt half-Poiseuille profile far upstream and downstream of the step. In particular, we found that taking $L=12$ is sufficient to obtain a converged profile to within graphical accuracy, and we adopt this value throughout the remainder of this section.

We now study in detail the limit $d \rightarrow 0$. Figure 2 shows the free-surface profiles for the various cases $d=0.5,0.2$, and 0.02 . The profiles appear to converge to a continuous limit as $d$ is reduced. This is also apparent from figure 3(a), which shows the behaviour of the maximum of $F$ for the same values of the parameters. Moreover, the results in figure 3 indicate that both the first and second derivatives of $F$ remain 


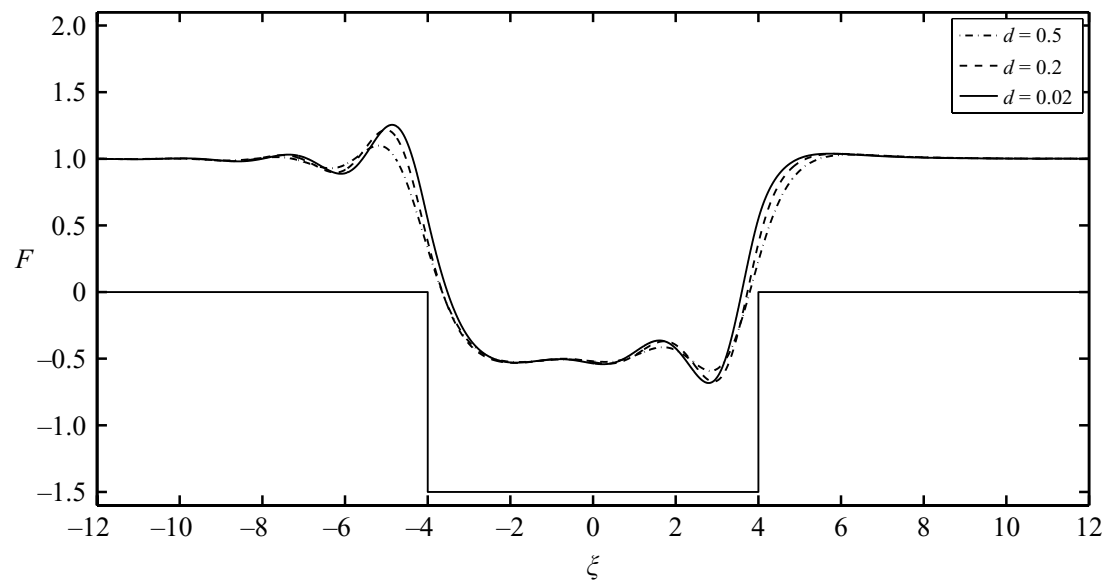

FIGURE 2. Steady-state profiles, $F(\xi)$, when $C^{\prime}=1$ and $W_{e}^{\prime}=1$, for flow over a trench with $D=0.75, B=4$, and $d=0.5$ (dot-dashed line), $d=0.2$ (dashed line), and $d=0.02$ (solid line). The computations were performed with $L=12$ and $M=3000$. The limiting rectangular wall profile is shown for illustrative purposes only.

(a)

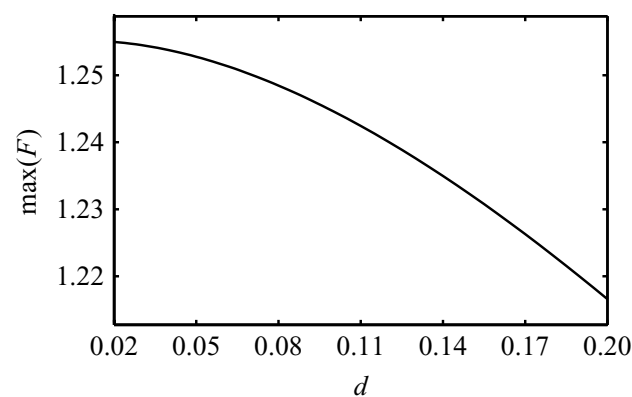

(c)

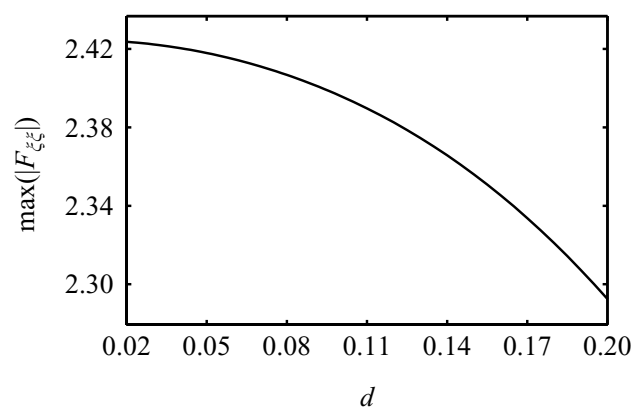

(b)

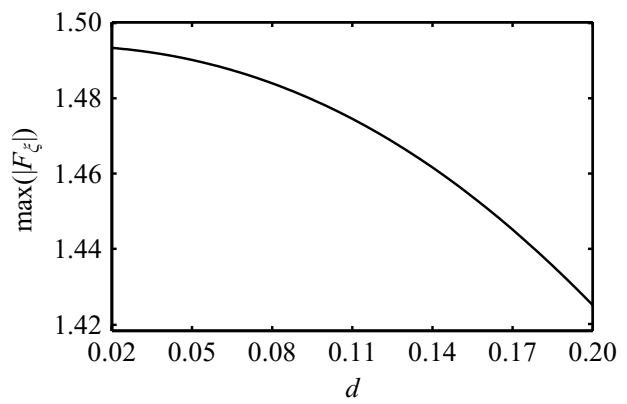

$(d)$

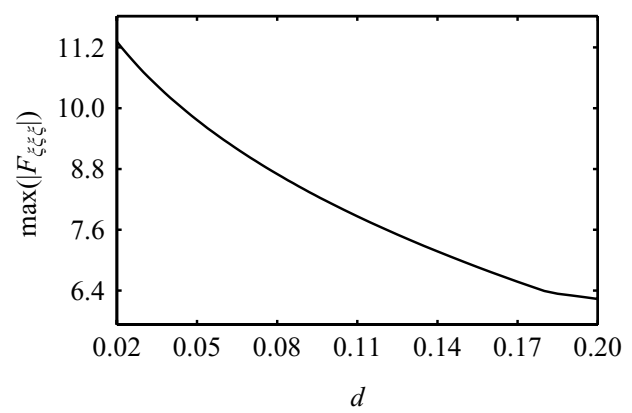

FIGURE 3. The variation of the maxima of $F,\left|F_{\xi}\right|,\left|F_{\xi \xi}\right|$, and $\left|F_{\xi \xi \xi}\right|$ with the steepness parameter $d\left(a, b, c\right.$ and $d$, respectively) for a trench with $D=0.75$ and $B=4$ when $C^{\prime}=1$ and $W_{e}^{\prime}=1$. The computations were performed with $L=12$ and $M=3000$.

bounded in the limit $d \rightarrow 0$, suggesting that the limiting profile is at least twice differentiable. The behaviour of the third derivative, $F_{\xi \xi \xi}$, also shown, is not so easy to discern; it may either remain bounded or else blow up as $d$ tends to zero. However, if the first two derivatives of $F$ are bounded, as seems to be the case, blow-up in $F_{\xi \xi \xi}$ 
(a)

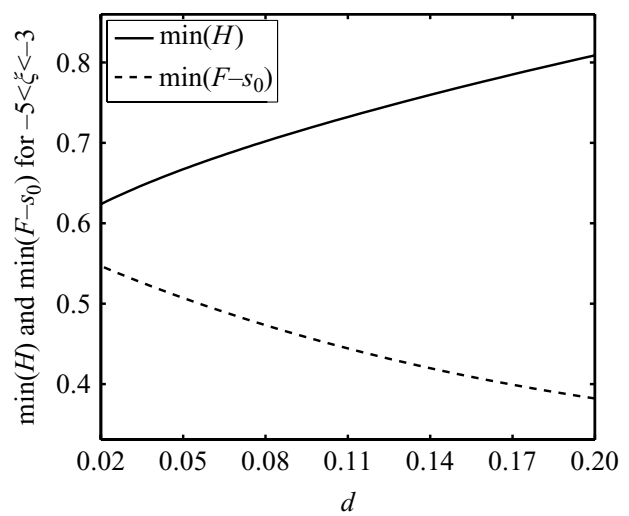

(b)

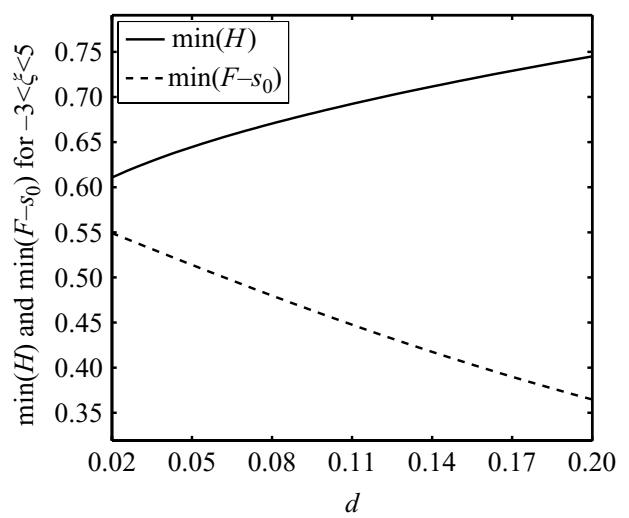

Figure 4. The variation of the local minima of the film thickness $H=F-s$ and of $F-s_{0}$ with the steepness parameter $d$ in the vicinity of the downward step $(a)$ and the upward step $(b)$ for a trench with $D=0.75$ and $B=4$ when $C^{\prime}=1$ and $W_{e}^{\prime}=1$. The calculations were carried out with $L=12$ and $M=3000$.

must be accompanied by blow-up in $P_{\xi}$, but we will present strong evidence below that the pressure gradient is in fact bounded.

Following these observations and making the physically plausible assumption that the free-surface shape $F$ is a continuous function of $\xi$, we conclude that the film thickness function $H=F-s$ must develop a jump discontinuity in the limit $d \rightarrow 0$ at each point where $s$ becomes discontinuous. Suppose that $s$ has a jump discontinuity at the point $\xi=\xi_{0}$. Then $H$ must also have a jump discontinuity at the same point. Steady-state solutions are determined by the steady version of (3.15), namely $\left[H^{3} P_{\xi}\right]_{\xi}=0$, where $P$ is given by (3.12). Integrating once gives $H^{3} P_{\xi}=C_{0}$, for some constant $C_{0}$. It follows that

$$
\lim _{\xi \rightarrow \xi_{0}^{-}}\left(H^{3} P_{\xi}\right)=\lim _{\xi \rightarrow \xi_{0}^{+}}\left(H^{3} P_{\xi}\right)=C_{0} .
$$

We now have the following possibilities.

(i) If both $H\left(\xi_{0}^{-}\right) \neq 0$ and $H\left(\xi_{0}^{+}\right) \neq 0$, then $P_{\xi}$ is bounded but has a jump discontinuity at $\xi=\xi_{0}$.

(ii) If either $H\left(\xi_{0}^{-}\right)=0$ or $H\left(\xi_{0}^{+}\right)=0$, then either $P_{\xi}\left(\xi_{0}^{-}\right)$is infinite or $P_{\xi}\left(\xi_{0}^{+}\right)$is infinite.

The second possibility corresponds to the case when the limiting film just touches the wall at the corner. For the first possibility the film remains at a finite distance from the wall all the way along its length.

To decide between the two options, we consider the minima of the film thickness over the length of the film as the parameter $d$ is reduced. For each value of $d$, we compute the free-surface profile, $F(\xi)$, over the smooth trench, $s(\xi)$, given by (4.5). Using this profile, we find the local minimum values of $H=F-s$ and $F-s_{0}$, where $s_{0}(\xi)$ is given by (4.4). Figure 4(a) displays the local minima obtained in the vicinity of the downward step, and figure $4(b)$ displays the local minima in the vicinity of the upward step. In both graphs, the minima of $F-s$ for the smooth step decrease monotonically as $d$ decreases, while the minima of $F-s_{0}$ for the sharp step increase monotonically as $d$ decreases. These results suggest that the minima will approach a non-zero limit as $d$ approaches zero, and that it is option (i) which is correct. By 


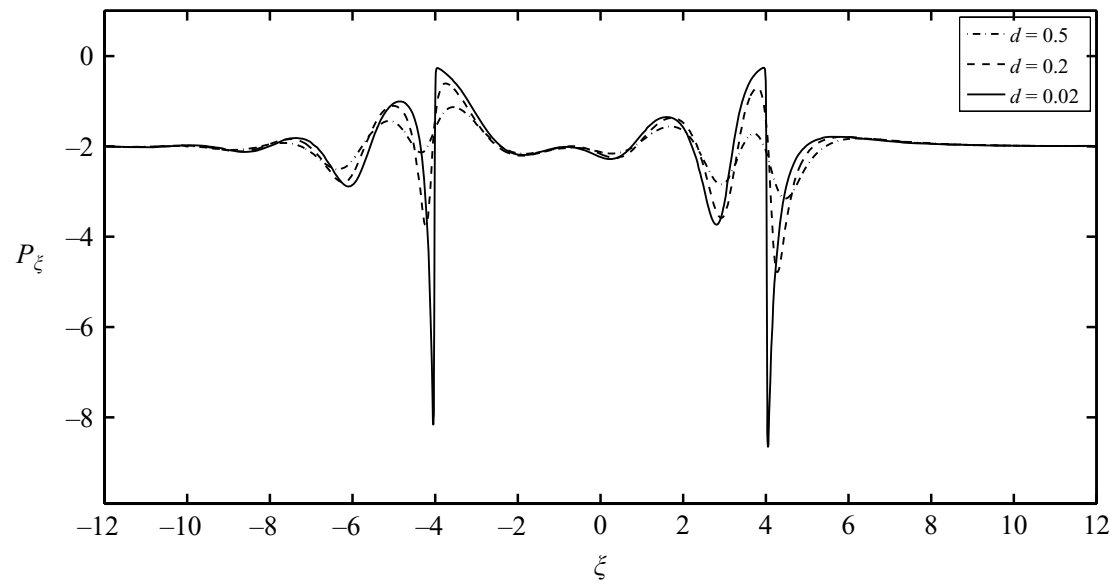

Figure 5. The dependence of the pressure gradient, $P_{\xi}$, on $\xi$ for a trench with $D=0.75, B=4$, and $d=0.5$ (dot-dashed line), $d=0.2$ (dashed line), and $d=0.02$ (solid line) when $C^{\prime}=1$ and $W_{e}^{\prime}=1$. The calculations were carried out with $L=12$ and $M=3000$.

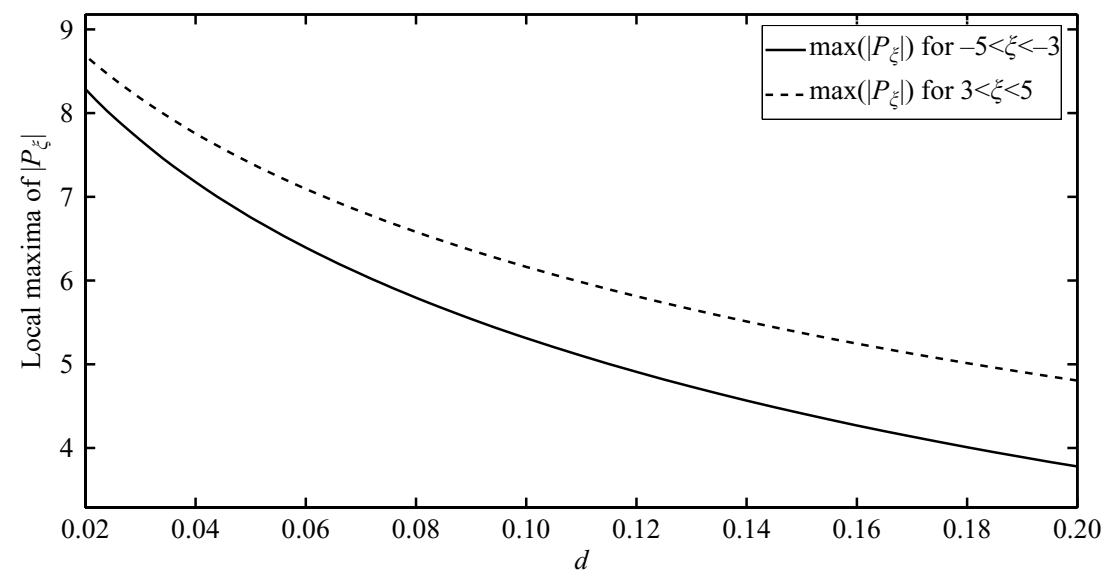

FIGURE 6. The variation of the local maxima of $\left|P_{\xi}\right|$ with the steepness parameter $d$ in the vicinity of the downward step (solid line) and the upward step (dashed line) for a trench with $D=0.75$ and $B=4$ when $C^{\prime}=1$ and $W_{e}^{\prime}=1$. The computations were performed with $L=12$ and $M=3000$.

extrapolation, we can obtain an estimate of 0.58 for both of the limiting values of the local minima at the downward and upward steps, that is $H(\xi) \geqslant 0.58$ for each $\xi$ in one period. From (4.8), it follows that $\left|P_{\xi}\right|$ is bounded above by $\left|C_{0}\right| / 0.58^{3}$. The constant $C_{0}$ can be estimated by considering the values of $H$ and $P_{\xi}$ at $\xi=-12$, for example. Profiles of $P_{\xi}$ are displayed in figure 5 for the cases $d=0.02,0.2$, and 0.5. The maximum local values of $\left|P_{\xi}\right|$ around the downward and upward steps are shown in figure 6 and appear to be increasing as $d$ decreases in apparent support of option (ii). However, given that $H(-12)=1$ and $P_{\xi}(-12)=-2$ (see figures 2 and 5), we can estimate that $C_{0} \approx-2$. Thus, $\left|P_{\xi}\right|$ is in fact bounded by $2 / 0.58^{3} \approx 10.25$. Moreover, $P_{\xi}$ appears to be developing jump discontinuities at the downward and upward steps, and our results support option (i), namely that the film remains a finite distance from the wall as $d$ tends to zero and the case of sharp right-angled steps is approached. 
The numerical estimate $C_{0} \approx-2$ can be placed on a firmer footing by the following analysis. Rewriting the governing equation $H^{3} P_{\xi}=C_{0}$ in the form

$$
\frac{C_{0}}{H^{3}}+2=A_{\xi}
$$

where $A(\xi)=-F_{\xi \xi} / C^{\prime}-2 W_{e}^{\prime} \mathscr{H}\left[F_{\xi}\right]$, and averaging over $[-L, L]$ gives

$$
C_{0}\left[\frac{1}{2 L} \int_{-L}^{L} \frac{1}{H^{3}} \mathrm{~d} \xi\right]+2=\frac{A(L)-A(-L)}{2 L} .
$$

Due to the periodicity of $F$, we get $A(L)=A(-L)$, and therefore the right-hand side of (4.10) vanishes. We can obtain an estimate for $C_{0}$ by considering the limit $L \rightarrow \infty$. This in turn gives a good approximation for $C_{0}$ in the numerical solutions, since $L$ is taken to be large there. Using the fact that $H \rightarrow 1$ as $|\xi| \rightarrow \infty$, we find that

$$
\lim _{L \rightarrow \infty} \frac{1}{2 L} \int_{-L}^{L} \frac{1}{H^{3}} \mathrm{~d} \xi=1 .
$$

Using this result in (4.10) we find that $C_{0} \rightarrow-2$ as $L \rightarrow \infty$, which is consistent with the numerical estimate.

\subsection{Film flow over a single step}

Flow over an isolated step is recovered by taking the double limit $L \rightarrow \infty$ and $B \rightarrow \infty$ in (4.5). In our calculations, we fix $D=1, d=0.2, L=30$, and $B=15$. The latter two values are found by experimentation to be sufficiently large to give accurate results for a single step. For example, we checked that the velocity profile in the film approaches the expected Nusselt half-Poiseuille profile far upstream and downstream of the step. In the following calculations, we fix the rescaled capillary number to be $C^{\prime}=1$ and vary the rescaled electric Weber number, $W_{e}^{\prime}$, to assess the effect of the electric field on the film profile.

Typical results for a downward/upward step are shown in figure $7(a, c, e, g)$ and figure $7(b, d, f, h)$, respectively. The four panels in each column show solutions for $W_{e}^{\prime}=0,1.5,3$, and 4.5 . The free-surface profile generally mimics that of the topography with some deviations in the neighbourhoods of the steps; moreover, these deviations can be controlled by the electric field. In the absence of an electric field, when $W_{e}^{\prime}=0$, the free surface develops a capillary ridge just upstream of the downward step. An electric field of low intensity introduces short-wave oscillations on the capillary ridge above the downward step as well as a small depression immediately downstream of the step. Increasing $W_{e}^{\prime}$ reduces the magnitude of the oscillations and the height of the capillary ridge, whilst also slightly deepening the depression beyond the step. As the electric field is increased further, the capillary ridge is completely eliminated. Figure 8 shows how the maximum and minimum values of the film height $F$ at the downward and upward steps vary with $W_{e}^{\prime}$. These are computed as the maximum and minimum values of $F$ over the range $-20 \leqslant \xi \leqslant-10$ at the downward step and $10 \leqslant \xi \leqslant 20$ at the upward step. For a downward step, both the maximum and minimum values of the film height are monotonically decreasing functions of $W_{e}^{\prime}$. Note that the depression beyond the downward step for large values of $W_{e}^{\prime}$ is less pronounced than the height of the capillary ridge found before a downward step in the absence of an electric field. It is also observed that the film is pulled away from the downward step as $W_{e}^{\prime}$ increases.

The opposite behaviour is found at an upward step. In this case, with no electric field present, a depression is formed right ahead of the step. Increasing the electric 
(a)

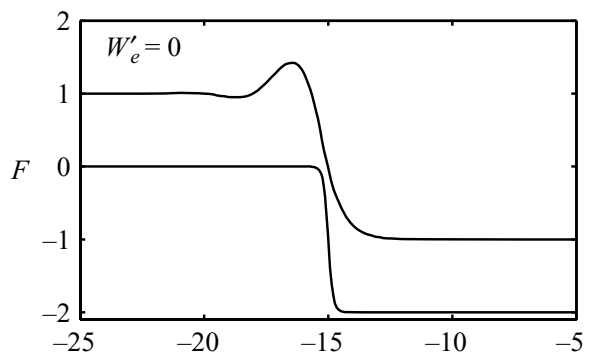

(c)

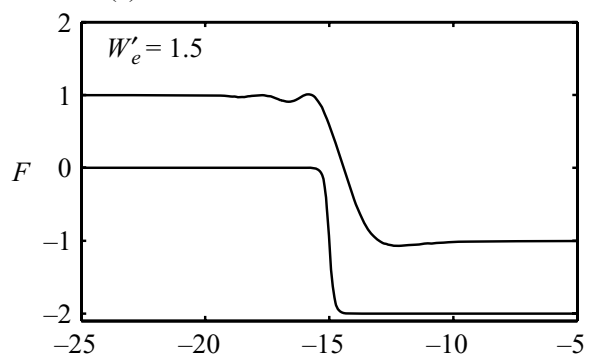

(e)

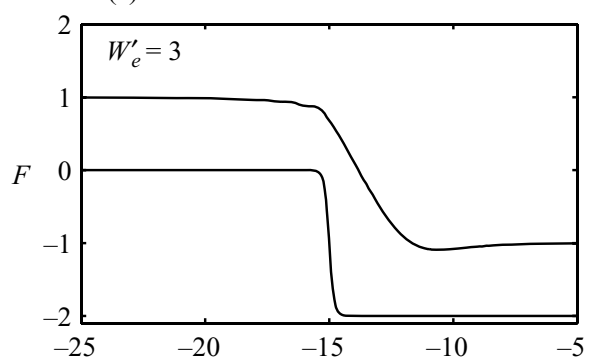

$(g)$

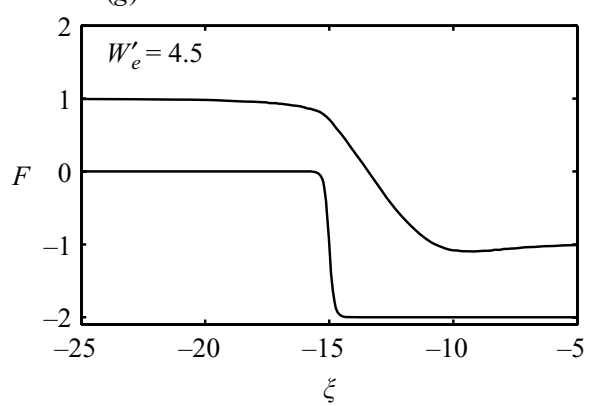

(b)

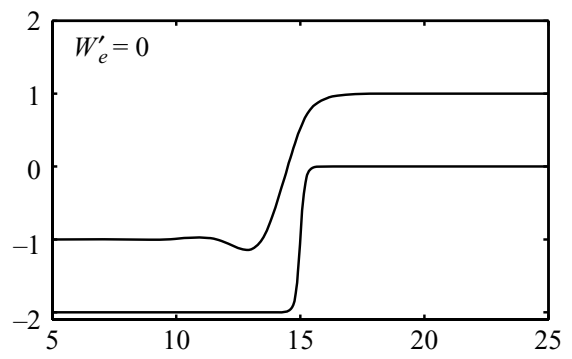

(d)

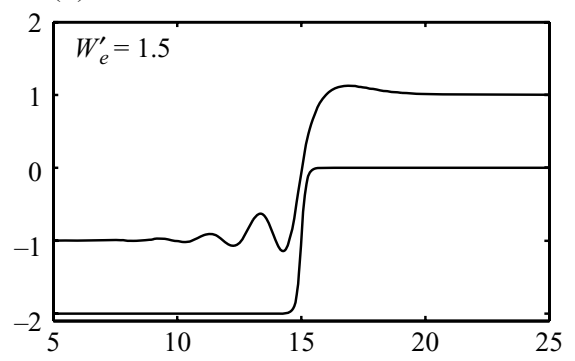

$(f)$

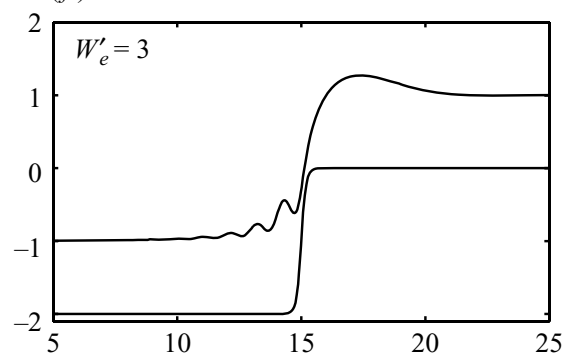

(h)

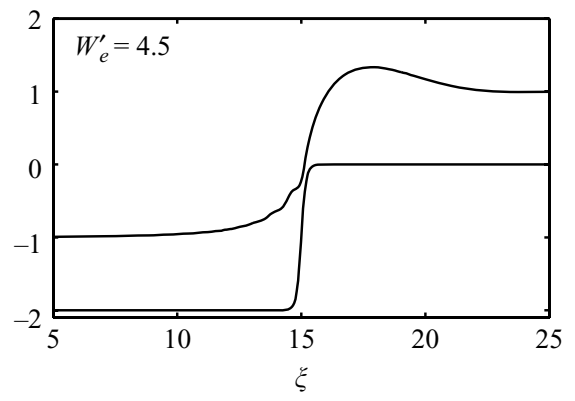

FIgURE 7. Steady-state profiles, $F(\xi)$, at a downward step $(a, c, e, g)$ and at an upward step $(b, d, f, h)$ for $C^{\prime}=1$ and $W_{e}^{\prime}=0,1.5,3$, and 4.5. For each step $B=15, D=1$, and $d=0.2$. The calculations were carried out with $L=30$ and $M=1500$.

field from zero introduces short-wave oscillations on the free surface in front of the step. As $W_{e}^{\prime}$ increases up to about 1.5 , the amplitude of the oscillations becomes quite large and the depression deepens. As $W_{e}^{\prime}$ is increased beyond 1.5, the amplitude of the short-wave oscillations is reduced and the depression is eliminated but a ridge now forms above the step, although it is less pronounced than that encountered at 
(a)

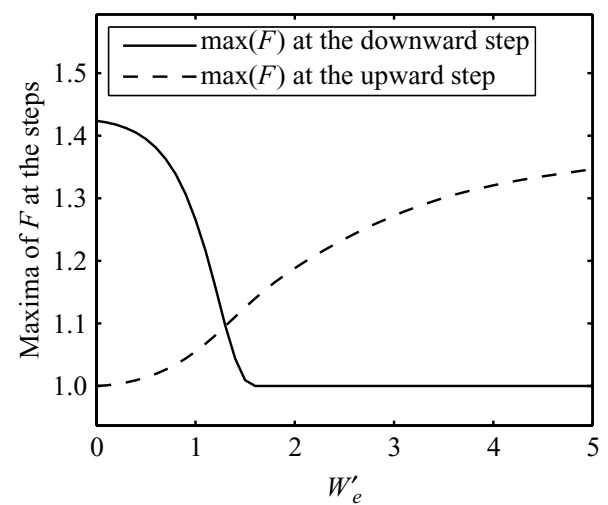

(b)

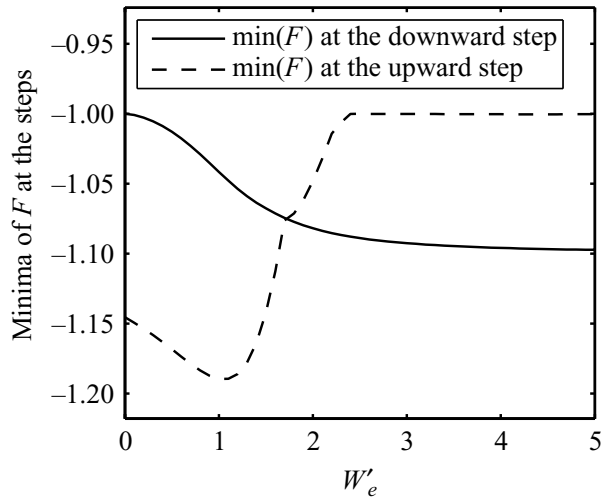

Figure 8. The maxima $(a)$ and minima $(b)$ of the steady film profile, $F(\xi)$, as functions of $W_{e}^{\prime}$ at a downward and an upward step for $C^{\prime}=1$. The equations were integrated with $L=30$ and $M=1500$ for steps with $B=15, D=1$, and $d=0.2$.

(a)

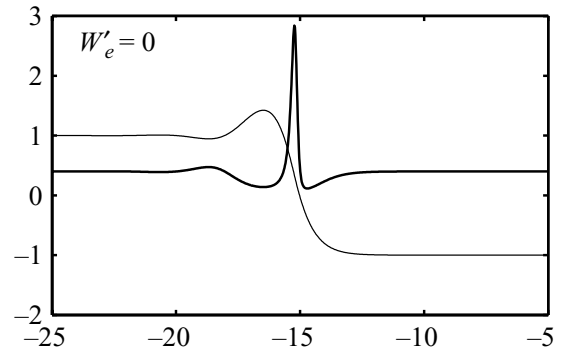

(c)

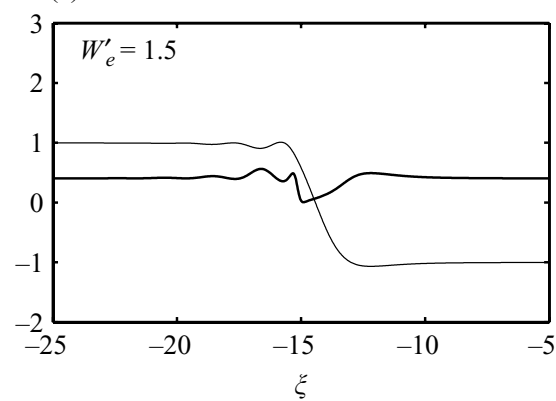

(b)

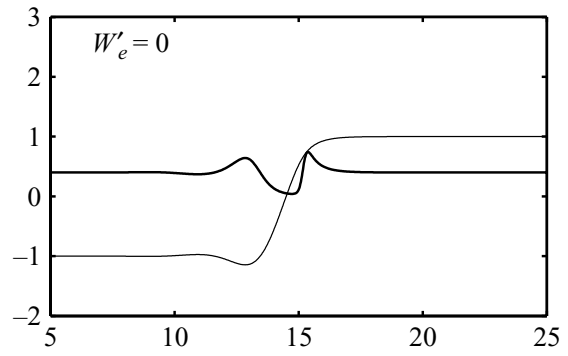

(d)

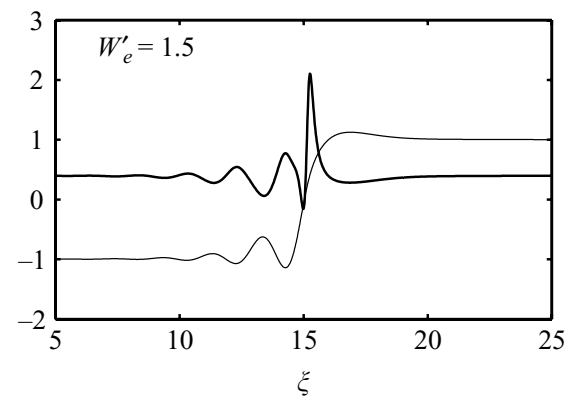

Figure 9. The scaled negative pressure gradient, $-P_{\xi} / 5$, shown as thick lines, together with the free-surface profiles, shown as thin lines, at a downward step $(a, c)$ and an upward step $(b, d)$ for $C^{\prime}=1$ and $W_{e}^{\prime}=0$ and 1.5. In each case $B=15, D=1$, and $d=0.2$ and the steps are centred at $\xi= \pm 15$. The calculations were carried out with $L=30$ and $M=1500$.

a downward step in the absence of an electric field. As the Weber number increases from zero, the film is pulled towards the step.

Some insight into the physical mechanisms responsible for the flow features is provided in figures 9 and 10 . In figure 9, the negative scaled pressure gradient, $-P_{\xi} / 5$, is plotted on top of the free-surface profiles at a downward step and at an upward step both with and without an electric field. In each graph, the pressure gradient is 
shown with a thick line, and the free-surface profile is shown with a thin line. The steps are at $\xi= \pm 15$. The $(a-d)$ panels correspond to figure $7(a-d)$. Considering the downward step with no electric field and looking from left to right, we note that the first very slight depression in the free-surface profile in front of the capillary ridge coincides with a rise in the negative pressure gradient, which drives fluid faster, thereby thinning the film to ensure mass conservation. Conversely, the capillary ridge is accompanied by a dip in the negative pressure gradient as fluid is slowed down before traversing the step. The negative pressure gradient then rises very sharply, pushing the fluid rapidly over the step. The increase in speed leads to a thinning of the film, which is particularly noticeable at the apex of the step, as can be seen in figure $7(a)$. When the electric field is switched on, the sharp rise in the negative pressure gradient over the step is eliminated, the fluid flows more sluggishly over the step, and the thinning effect is much less pronounced. For the upward step, the electric field has the opposite effect. It provokes a much larger negative pressure gradient over the step than would otherwise be present and speeds up the fluid just before it climbs up the step. The fluid must be slowed down again to permit the film height to return to its undisturbed upstream value. This explains the presence of the ridge just beyond the step, where the negative pressure gradient dips and the fluid flow is retarded.

To explain the fluctuations in the pressure gradient, we begin by integrating the governing equation (4.1) with respect to $\xi$ and arranging the result into the form

$$
\frac{C_{0}}{H^{3}}+2+\frac{1}{C^{\prime}} H_{\xi \xi \xi}+2 W_{e}^{\prime} \mathscr{H}\left[H_{\xi \xi}\right]=-\frac{1}{C^{\prime}} s_{\xi \xi \xi}-2 W_{e}^{\prime} \mathscr{H}\left[s_{\xi \xi}\right] .
$$

This is analogous to the forced equation (6a) of Kalliadasis et al. (2000) excluding electric field effects. In the absence of topographical forcing, the right-hand side is zero, the film is purely flat, $H=1$, and the pressure gradient is uniform. Deviations from a flat film in the presence of topography may, therefore, be understood in terms of the forcing effects on the right-hand side. In figure 10, we plot the two forcing contributions $s_{\xi \xi \xi} / C^{\prime}$ and $2 W_{e}^{\prime} \mathscr{H}\left[s_{\xi \xi}\right]$ together with the wall profiles at a downward step and at an upward step. The arrows indicate the direction in which the force is pushing fluid. The sizes of the forcing terms have been scaled down for illustrative purposes. It is noticeable that the general shapes of the forcing curves are qualitatively similar to the pressure gradient profiles in figure 9, except for some oscillations in the presence of an electric field. Concentrating first on the effect of the topography, seen in the upper two panels, we see that the fluid is forced backwards just before the step. This explains the local dip in the pressure gradient and also the creation of the capillary ridge. Fluid is forced rapidly over the step before again being pushed backwards at the bottom in order to slow down the fluid and enable the film to return to its original undisturbed height upstream. In contrast, the topography forces fluid onwards at the foot of the upward step, creating the small depression seen in figure $7(b)$. The fluid is slowed before mounting the step, in line with physical intuition, and is then accelerated forwards at the top of the step to return the film to its original undisturbed height downstream.

The role played by the electrical forcing term is seen in figure $10(c, d)$. The electrical forcing pushes fluid downstream just above the downward step, thereby weakening the upstream acceleration imparted by the topography and reducing the amplitude of the capillary ridge. It slows the fluid passing over the step, causing a thickening of the film and effectively pulling the film away from the apex, in agreement with what was observed in figure $7(e, g)$. Just beyond the step, a slight positive electrical forcing 
(a)

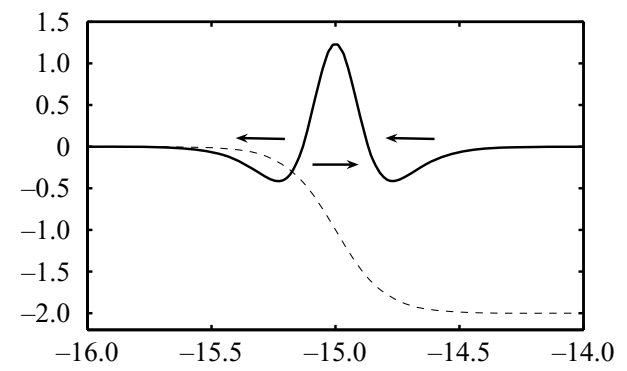

(c)

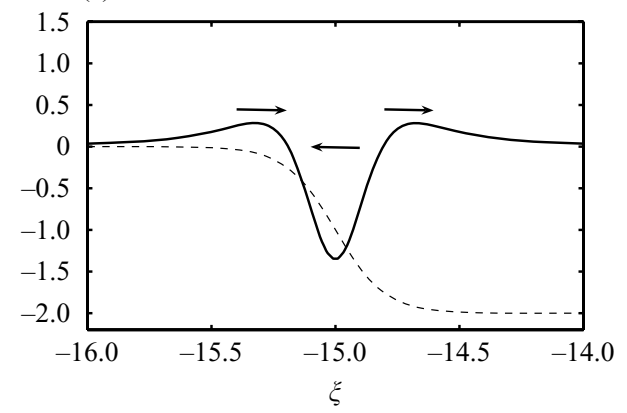

(b)

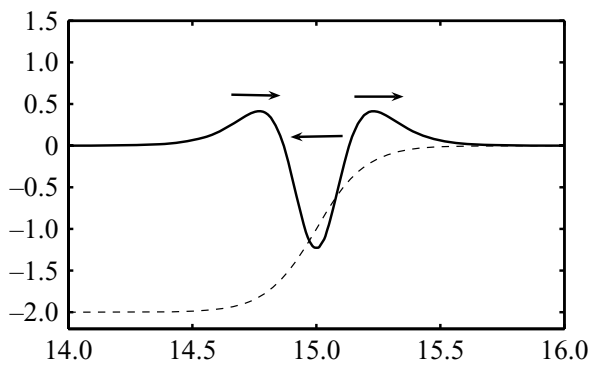

$(d)$

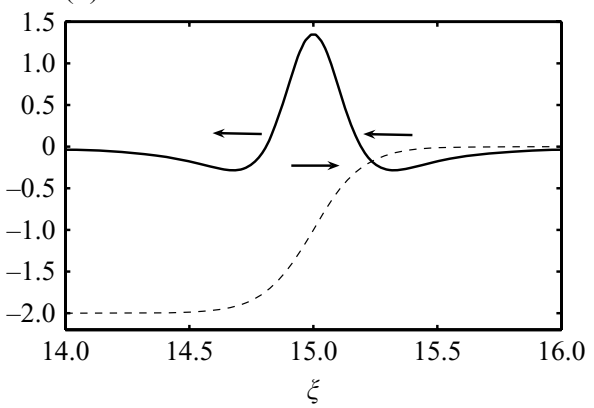

Figure 10. The forcing effects at a downward step $(a, c)$ and an upward step $(b, d)$. The topographical forcing term $(a, b), s_{\xi \xi \xi} / C^{\prime}$, and the electrical forcing term $(c, d), 2 W_{e}^{\prime} \mathscr{H}\left[s_{\xi \xi}\right]$, are shown as solid lines overlaid onto the wall profile, shown as broken lines. In each case the size of the forcing terms has been scaled to fit the graph for ease of illustration. For all panels, $D=1$ and $d=0.2$ and the steps are centred at $\xi= \pm 15$. The arrows indicate the direction in which the force is pushing fluid.

pushes fluid faster downstream generating the slight depression seen in the profiles in figure 7. As $W_{e}^{\prime}$ increases, the depression deepens. At an upward step, a positive electrical forcing pushes the fluid faster over the step, thereby thinning the film, as seen in figure $7(f, h)$. A slightly negative electrical forcing beyond the step attempts to push fluid back upstream, and this explains the appearance of the ridge. As $W_{e}^{\prime}$ increases, the amplitude of the ridge grows.

The forcing effects just described explain the basic observed features, such as the emergence of ridges and depressions. However, secondary features, such as short-wave oscillations showing up when an electric field is turned on, are not explained by this analysis.

The effect of varying the depth of the downward step or varying the height of an upward step is shown in figure 11. Panels $(a)$ and $(b)$ show profiles obtaining in the absence of an electric field, $W_{e}^{\prime}=0$, and these should be compared with figures $3(a)$ and 3 (b) of Kalliadasis et al. (2000). As $D$ is increased for a downward step, the amplitude of the capillary ridge increases monotonically. Similarly, the depression preceding the ridge grows deeper as $D$ increases. For an upward step, increasing $D$ has virtually no effect on the downstream profile, but it tends to deepen the depression seen just before the step and also shift it slightly upstream. These observations change markedly in the presence of an electric field, as can be seen in figure 11 $(c, d)$. For example, at a downward step with $W_{e}^{\prime}=1.2$, as $D$ increases, the amplitude of the capillary ridge first increases, then decreases, and then increases again. Also, the slight depression on the downstream side of the step becomes more noticeable with increasing $D$. For the 
(a)

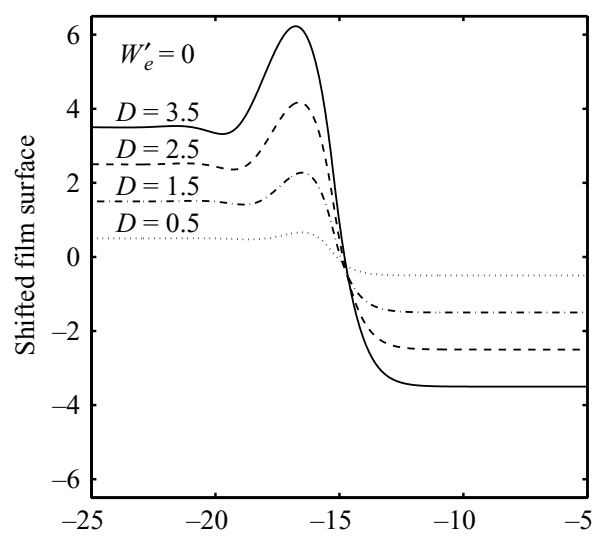

(c)

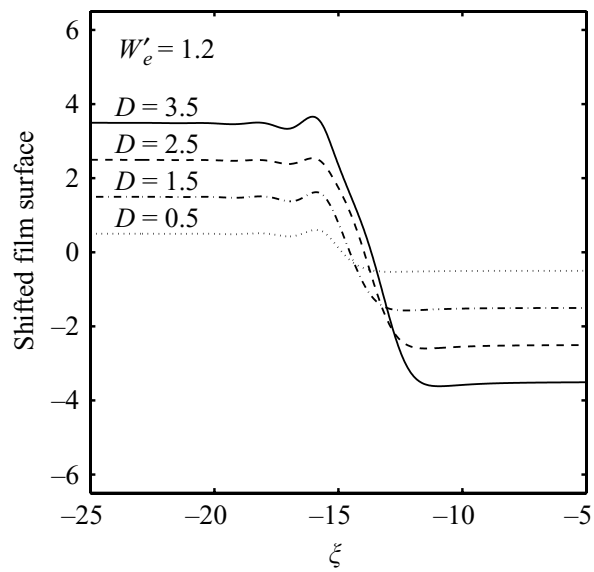

(b)

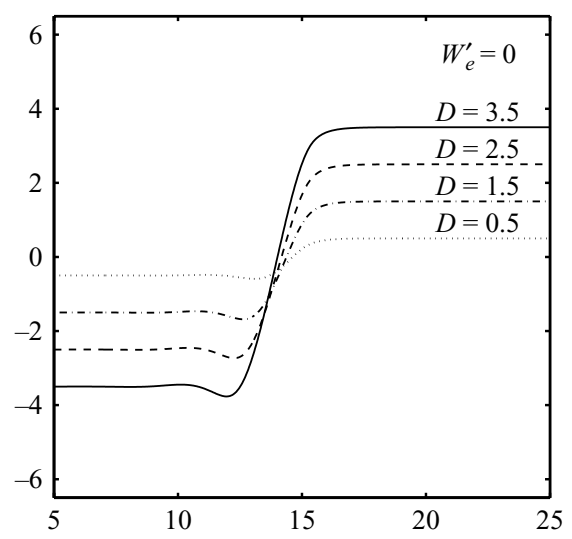

(d)

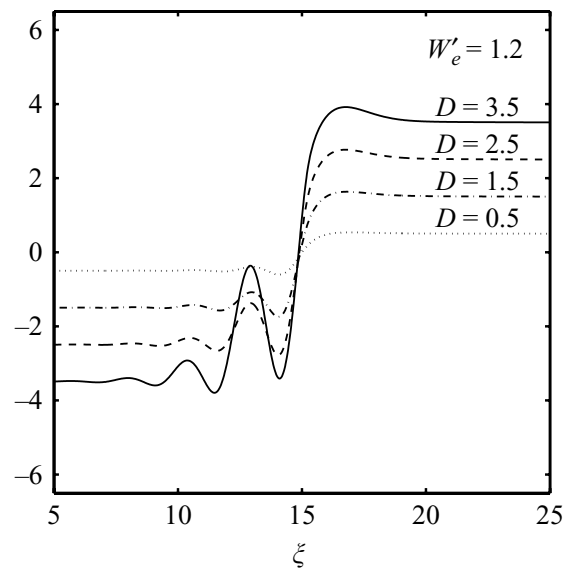

FIGURE 11. The effect of varying the depth, $D$, of a downward step $(a, c)$ and an upward step $(b, d)$ for $W_{e}^{\prime}=0(a, b)$ and $W_{e}^{\prime}=1.2(c, d)$ when $C^{\prime}=1$. The profiles have been shifted upwards for illustrative purposes. The equations were integrated with $L=30, B=15, d=0.2$, and $M=1500$.

upward step, increasing $D$ now has a significant effect on the downstream profile with the creation of a ridge of increasing amplitude. Moreover, the profile just upstream of the step develops more severe oscillations as $D$ increases.

\subsection{Film flow into a trench and over a mound}

To examine flow into trenches and over mounds of finite breadth, we fix $B$ and take $L$ to be large to ensure unidirectional oncoming flow. We found that taking $L=30$ is sufficient to obtain accurate results. In the following calculations, we take $D=1$, $d=0.1$, and fix $C^{\prime}=1$ and $W_{e}^{\prime}=1.2$.

Results for flows into a trench and over a mound are shown in figures $12(a, c, e)$ and $12(b, d, f)$ for the cases $B=0.5,2$, and 4 . When $B=4$, the flow into a trench exhibits a capillary ridge just before a downward step followed by a shallow depression, as is found for the isolated downward step shown in figure $7(a, c, d, g)$. The film develops oscillations before leaving the trench and a slight elevation above the upward step, as is observed for the isolated upward step seen figure $7(b, d, f, h)$. In a similar way, the flow over a mound features oscillations just before the upward step, with 
(a)

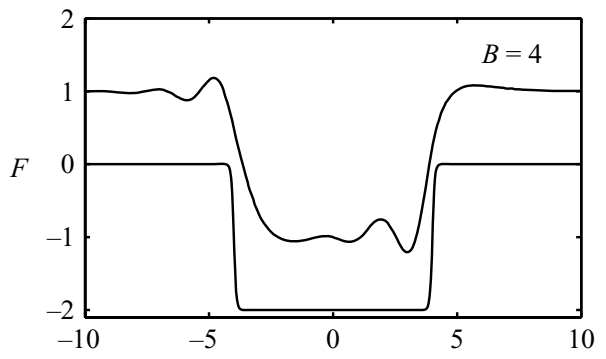

(c)

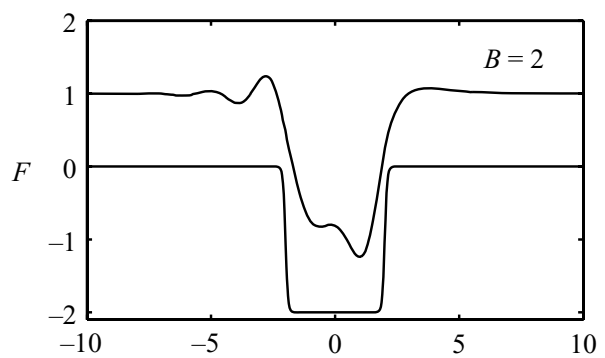

(e)

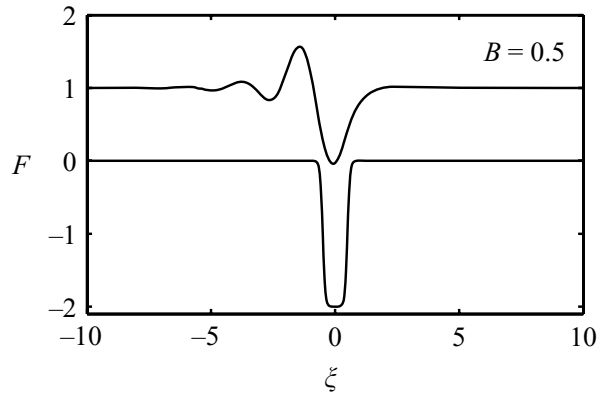

(b)

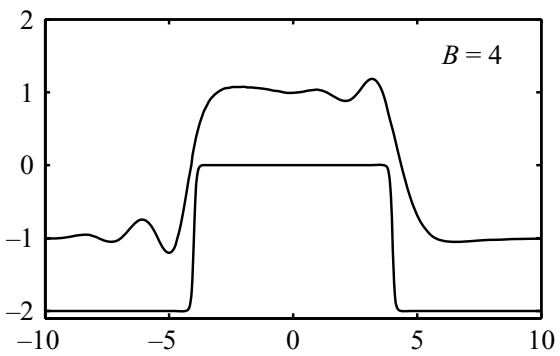

$(d)$

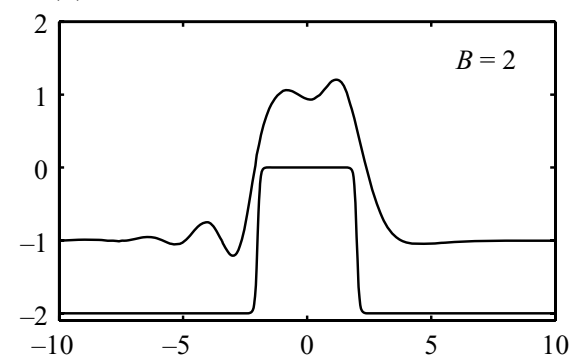

$(f)$

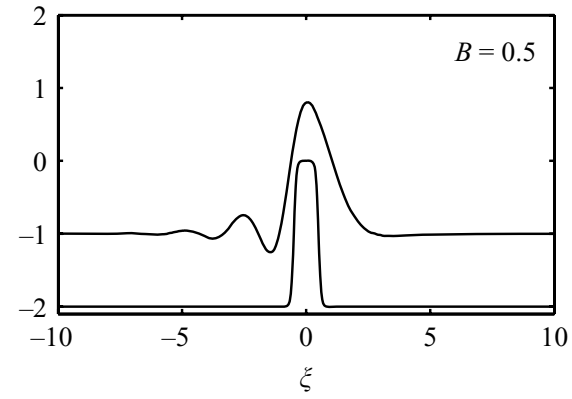

Figure 12. Steady-state profiles, $F(\xi)$, for flow into a trench $(a, c, e)$ and over a mound $(b, d, f)$ for $C^{\prime}=1$ and $W_{e}^{\prime}=1.2$. The wall parameters are $D=1$ and $d=0.1$, with $B=4$ $(a, b), B=2(c, d)$, and $B=0.5(e, f)$. The equations were integrated with $L=30$ and $M=2000$.

a slight elevation occurring above the step. On the downstream side of the mound, a capillary ridge precedes the downward step followed by a slight depression just beyond the mound. As $B$ decreases, the two profiles at the downward and upward steps start to interact. For trench flow, the disturbance to the free surface becomes less pronounced as the film is pulled up out of the trench. The height of the ridge above the downward step increases, while the slight elevation above the upward step is reduced. For mound flow, the capillary ridge on the downstream side of the mound disappears, leaving the free surface with a single hump, whose height decreases as $B$ decreases. The oscillations immediately upstream of the mound are little affected as the breadth of the mound decreases.

The influence of an electric field on film flow over a narrow trench or a narrow mound is shown in figures $13(a, c, e, g)$ and $13(b, d, f, h)$ for the case $L=30$, $B=0.5, D=1, d=0.1, C^{\prime}=1$, and for different values of $W_{e}^{\prime}$. As can be seen, film flow into a narrow trench in the absence of an electric field, with $W_{e}^{\prime}=0$, features a 
(a)

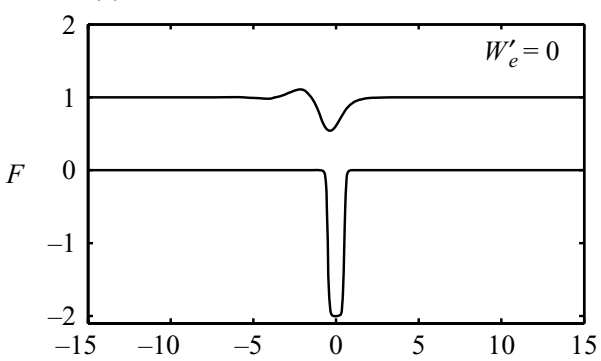

(c)

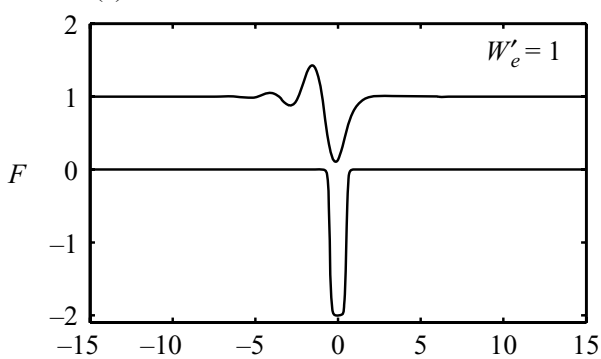

(e)

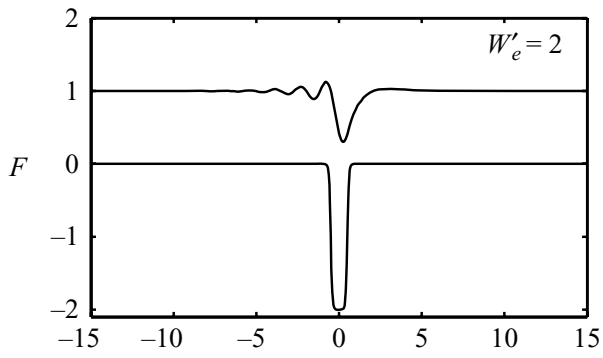

$(g)$

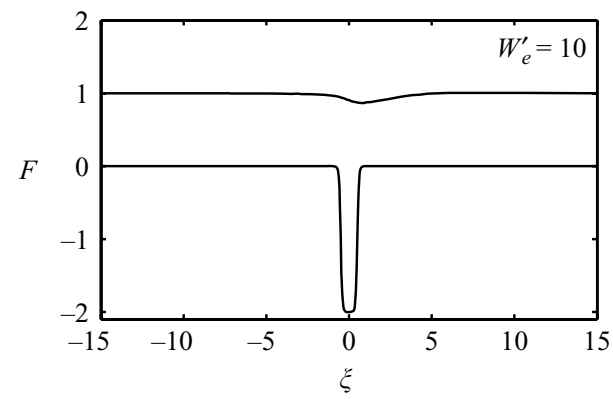

(b)

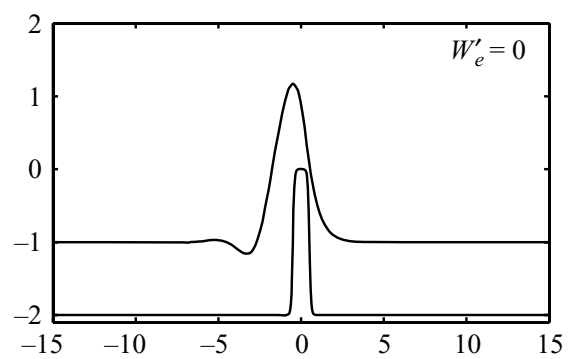

(d)

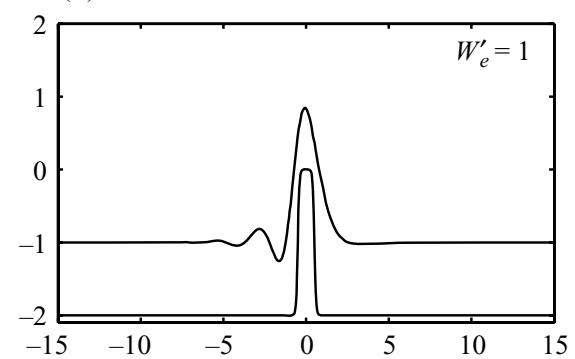

$(f)$

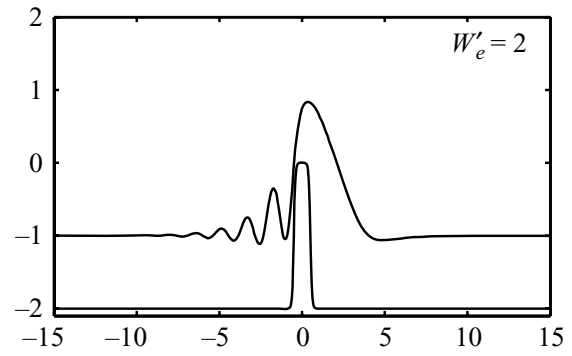

(h)

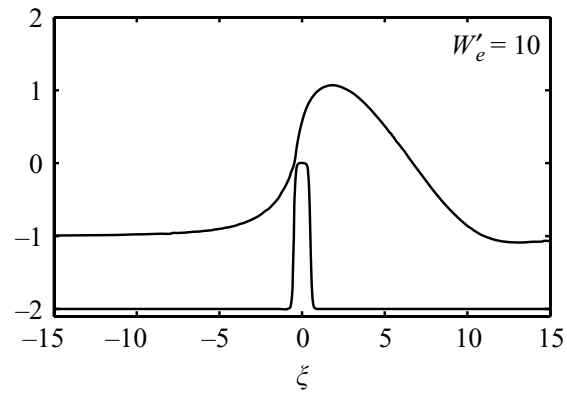

FIGURE 13. Steady-state profiles, $F(\xi)$, for flow into a narrow trench $(a, c, e, g)$ and over a narrow mound $(b, d, f, h)$ for $C^{\prime}=1$ and $W_{e}^{\prime}=0(a, b), W_{e}^{\prime}=1(c, d), W_{e}^{\prime}=2(e, f)$, and $W_{e}^{\prime}=10(g, h)$. The wall parameters are $B=0.5, D=1$, and $d=0.1$. The computations were performed with $L=30$ and $M=2000$.

capillary ridge just ahead of the trench followed by a trough, which moves further along the trench as the intensity of the electric field is increased. As $W_{e}^{\prime}$ increases, the height of the ridge and the depth of the trough start to grow, and short-wave oscillations appear on the free surface around the capillary ridge. When $W_{e}^{\prime}$ is further increased, the height of the ridge and the depth of the trough both decrease, and the 
amplitude of the short-wave oscillations is reduced. At larger values of $W_{e}^{\prime}$, the free surface is rendered almost flat but with a slight depression over the downstream edge of the trench. For flow over a narrow mound, it can be seen that when $W_{e}^{\prime}=0$, the free surface has a small depression before the mound followed by a pronounced peak over the mound. As $W_{e}^{\prime}$ is increased, the height of the peak goes down, the depth of the depression starts to grow, and short-wave oscillations appear localized around the depression. As $W_{e}^{\prime}$ is increased further, the peak rises and shifts downstream, and the short-wave oscillations increase in amplitude. As $W_{e}^{\prime}$ is increased still further, the free surface develops a single relatively thick hump downstream of the mound followed by a mild depression.

\subsection{Film flow down a slightly inclined wall}

When the substrate is inclined at only a very slight angle, the assumption adopted heretofore that $\cot \beta \ll \delta^{-1}$ could be violated. In particular, when $\beta=O(\delta)$ then $\cot \beta=O\left(\delta^{-1}\right)$ and the $z$-component of the gravitational force may no longer be neglected. In this case, a straightforward extension of the asymptotic analysis presented in $\S 3$ yields the modified equation for the steady-state film thickness,

$$
\left[\frac{2}{3} H^{3}-\frac{2 G}{3} H^{3}(H+s)_{\xi}+\frac{1}{3 C^{\prime}} H^{3}(H+s)_{\xi \xi \xi}+\frac{2}{3} W_{e}^{\prime} H^{3} \mathscr{H}\left[(H+s)_{\xi \xi}\right]\right]_{\xi}=0,
$$

where $G=\delta \cot \beta$. When $G=0$, this equation reduces to (4.1). An equivalent equation valid in the absence of an electric field was given by Kalliadasis et al. (2000), who used a slightly different non-dimensionalization. They found that increasing $G$ tends to eliminate the capillary ridge at a downward step and to eliminate the depression just upstream of an upward step. Solutions to (4.13) at a downward and an upward step are shown in figure 14. Panels $(a)$ and $(b)$ show results when there is no electric field, $W_{e}^{\prime}=0$. As $G$ increases, the amplitude of the capillary ridge at the downward step decreases monotonically. Similarly, increasing $G$ tends to monotonically reduce the depth of the depression found in front of the upward step. These observations are in line with those of Kalliadasis et al. (2000). Panels $(c)$ and $(d)$ show results in the presence of an electric field with $W_{e}^{\prime}=1.5$. In this case, the effect of increasing $G$ is ultimately the same but now the behaviour is non-monotonic. For example, as $G$ increases the amplitude of the short-wave oscillations on the capillary ridge at a downward step first increases and then decreases. Similarly, as $G$ increases the amplitude of the ridge above the upward step first increases and then decreases, while the oscillations just upstream of the step decrease in amplitude monotonically. In both panels $(a, b)$ and $(c, d)$, as $G$ increases the film is pulled towards the downward step and is pulled away from the upward step.

The observed trends can be broadly explained by reference to the new forcing contribution, $-2 G s_{\xi}$, which is identified by first rearranging (4.13) into a form equivalent to (4.12). This term is plotted in figure 15 at both a downward and an upward step. For a downward step, the effect of the new term is to help to push fluid downstream. This weakens the upstream acceleration imparted by the topographical forcing term just above the step (see figure 10a) and therefore acts to dampen the capillary ridge. Also, when $W_{e}^{\prime} \neq 0$, it strengthens the downstream acceleration imparted by the electrical forcing just downstream of the step (see figure 10c), and thereby supports the formation of the depression. Overall, it accelerates fluid over a downward step, thinning the film and effectively pulling it towards the step. Conversely, at an upward step the new gravitational forcing pushes fluid upstream. 

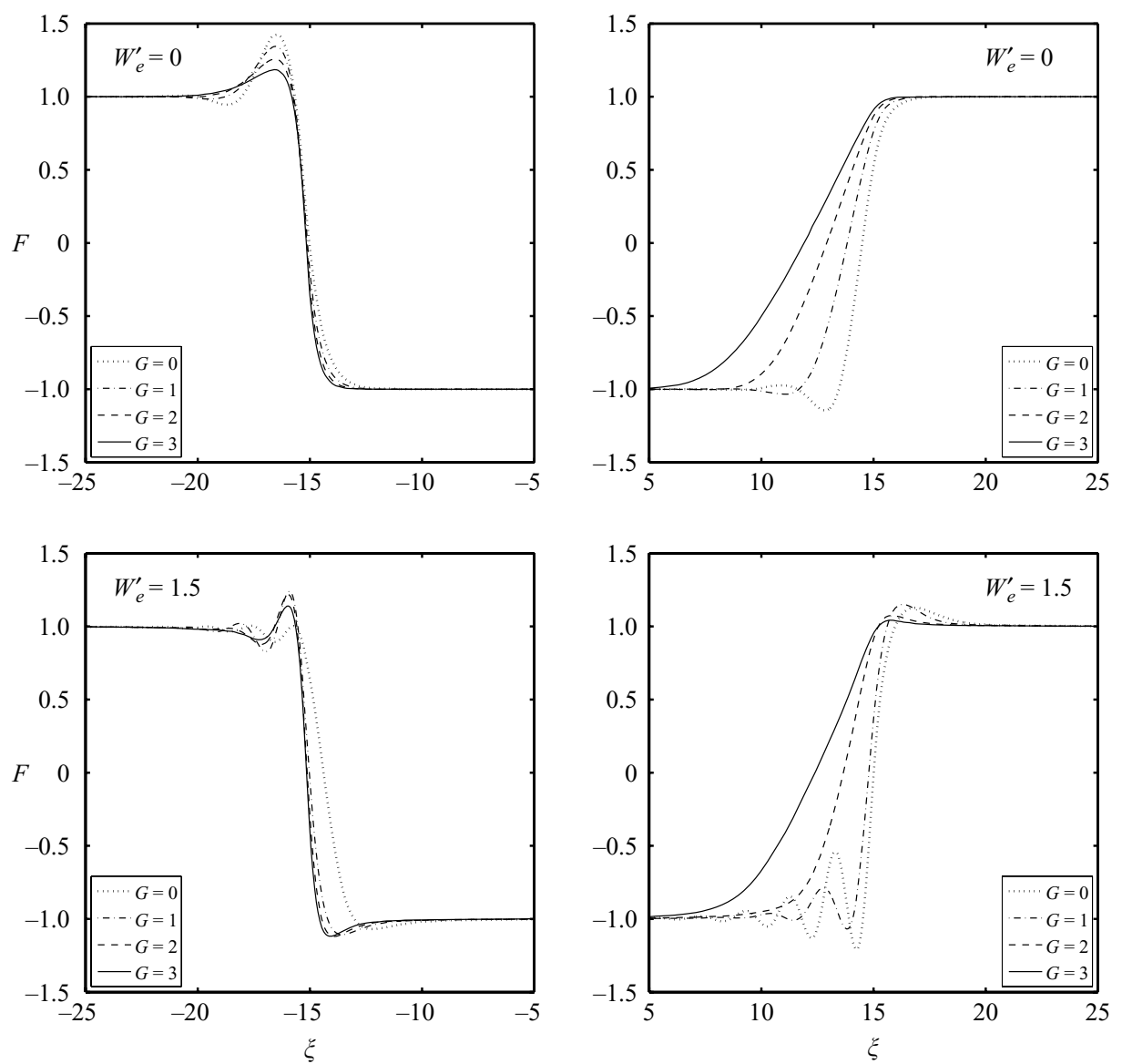

FIGURE 14. The effect of varying the 'gravitational' parameter, $G$, for a downward step $(a, c)$ and an upward step $(b, d)$ for $W_{e}^{\prime}=0(a, b)$ and $W_{e}^{\prime}=1.5(c, d)$. The equations were integrated with $L=30, B=15, D=1, d=0.2$, and $M=1500$.
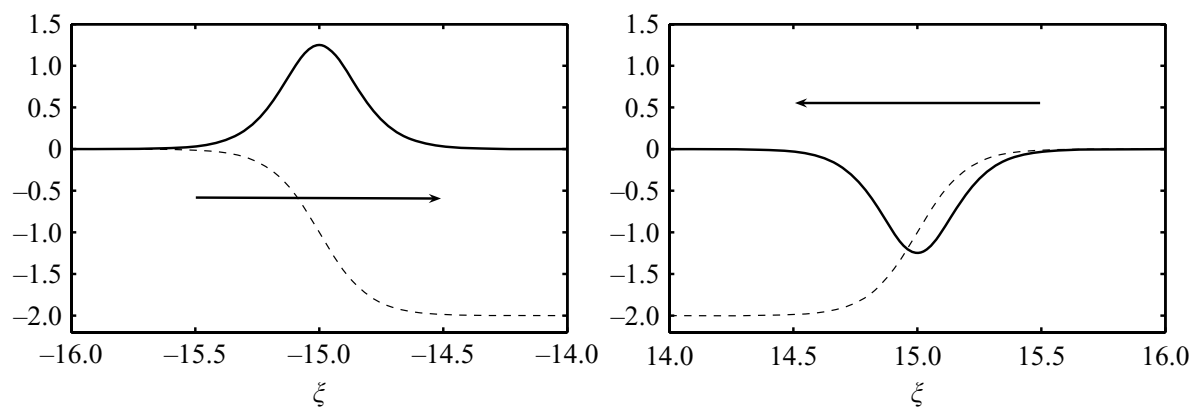

FIGURE 15. The forcing effect due to the $z$-component of gravity at a downward step $(a)$ and an upward step $(b)$. The forcing term, $-2 G s_{\xi}$, is shown as a solid line overlaid onto the wall profile, shown as a broken line. The size of the forcing term has been scaled to fit the graph for ease of illustration. For both $(a)$ and $(b), B=15, D=1$, and $d=0.2$ and the steps are centred at $\xi= \pm 15$. The arrows indicate the direction in which the force is pushing fluid. 
This weakens the downstream acceleration imparted by the topographical forcing term just upstream of the step (see figure $10 b$ ) and opposes the formation of the depression. Also, when $W_{e}^{\prime} \neq 0$, it strengthens the upstream acceleration imparted by the electrical forcing term just above the step (see figure 10); this explains why the amplitude of the ridge above the upward step can grow with increasing $G$ in the presence of an electric field. Overall, the new forcing effect decelerates fluid passing over an upward step, thickening the film and effectively pulling it away from the step.

\subsection{Physical parameter values}

To gain some insight into the physical relevance of our results, we consider typical values of our dimensionless parameters which might be realized in the laboratory. Since water acts as a near-perfect conductor, we take as a benchmark the experiments of Decré \& Baret (2003) on an aqueous film flowing under gravity down a wall indented with a rectangular trench in the absence of an electric field. The density, viscosity, and surface tension were measured at a temperature of $20^{\circ} \mathrm{C}$ to be

$$
\rho=10^{3} \mathrm{~kg} \mathrm{~m}^{-3}, \quad \mu=10^{-3} \mathrm{Pas}, \quad \gamma=7 \times 10^{-2} \mathrm{~N} \mathrm{~m}^{-1} .
$$

The undisturbed uniform film thickness upstream of the trench ranged between $80 \mu \mathrm{m}$ and $120 \mu \mathrm{m}$. The wall was at an angle of $\beta=30^{\circ}$ to the horizontal. Identifying $h_{0}$ with the undisturbed film thickness upstream, the capillary number, $C$, defined in (2.13), is found to be $C=2.24 \times 10^{-4}$ when $h_{0}=80 \mu \mathrm{m}$. Taking $C^{\prime}=1$, as in many of our calculations, gives a value of $\delta=0.06$. To obtain a rescaled electric Weber number of $W_{e}^{\prime}=0.1$, an electric field of strength $E_{0}=1.10 \times 10^{6} \mathrm{~V} \mathrm{~m}^{-1}$ is required. Assuming an electrode potential of $500 \mathrm{~V}$, this requires a gap between the film and the electrode of $456 \mu \mathrm{m}$, which is nearly 6 times the film height. This may well be sufficiently large for our analysis, which assumes that the electrode is a long way from the film, to hold. Also, the electric field strength lies below the critical value for dielectric breakdown of air, $3 \times 10^{6} \mathrm{~V} \mathrm{~m}^{-1}$, and so the configuration should be realizable in the laboratory.

\section{Discussion}

We have investigated the effect of a normal electric field on two-dimensional, gravity-driven flow of a perfectly conducting liquid film over topography. Working within the context of lubrication theory, we derived a nonlinear partial differential evolution equation for the film height. Subsequent attention was focused on steadystate, periodic solutions of this equation for flow over a downward step, an upward step, into a rectangular trench, and over a rectangular mound. In each case, the topography was approximated by a smoothly varying function, which allowed the corners to become increasingly sharp as a controlling parameter approaches zero. A careful analysis of the film behaviour in the limit of a wall with sharp right-angled corners provided strong evidence that the correct limiting configuration is one in which the film profile always remains at a finite distance from the wall. The limiting film profile is described by a twice continuously differentiable function whose third derivative has jump discontinuities at the points where the wall shape function is discontinuous.

In the absence of an electric field, previous studies have shown that the film profile develops a capillary ridge above a downward step and a slight depression just upstream of an upward step. The present results have revealed that an electric 
field is able to completely eliminate the capillary ridge. As a side effect, a slight depression appears immediately downstream of the downward step. Also, increasing the intensity of the electric field results in the film being pulled further away from the step as it passes over it. For upward steps, the opposite behaviour is found. In this case, the fluid film is pulled towards the step as the electric field is increased. The electric field can be used to eliminate the depression in front of an upward step. The side effect is that a slight ridge is introduced immediately above the step.

The effect of an electric field on film flow over narrow trenches and narrow mounds has also been examined. For the flows into narrow trenches, we have observed that in the absence of an electric field the free surface develops a capillary ridge just in front of the upstream edge of a narrow trench followed by a small trough. Increasing the electric field almost flattens the free surface, leaving only a slight depression over the trench. For film flow over a narrow mound, our results have shown that in the absence of an electric field the free surface features a pronounced peak over the mound preceded by a slight depression. When the electric field is increased, the peak widens into a hump and is shifted downstream. The slight depression before the mound is ironed out and a new depression appears beyond the hump.

When the inclination angle is sufficiently small, the governing equation includes an extra term reflecting the effect of the normal component of gravity. With no electric field, previous studies showed that the effect of this term is to smooth out undesirable free-surface features such as the capillary ridge. Our results have shown that this term can work in unison with electric field effects to amplify the deformation of the free surface.

Generally speaking, an electric field can either reduce or promote irregularities in the film surface depending on the local geometry. It is capable of removing the capillary ridge found at a downward step but leads to the creation of a ridge just above an upward step. As was described in the Introduction, previous works by Mazouchi \& Homsy (2001) and Gaskell et al. (2004) showed that the type of longwave theory adopted here is still capable of predicting the essential characteristics of the film profile over steep topographical features. We note, however, that it is unable to predict accurately the flow inside the film which will inevitably involve eddies inside a trench, for example. Boundary-integral calculations for Stokes flow, incorporating the effect of an electric field, will lend further credence to the film surface profiles presented here, and will, in addition, be able to predict flow features such as eddies. These calculations are already underway.

The present work has concentrated on computing steady solutions for electrified film flow, and we have said nothing about the stability of these solutions. Kalliadasis \& Homsy (2001) and Davis \& Troian (2005) examined the stability of the capillary ridge encountered at a downward step to spanwise perturbations in the absence of an electric field. Kalliadasis \& Homsy's results showed that the ridge is stable for a wide range of the pertinent parameter values. Linear stability analysis has shown that the effect of an electric field on a flat film is generally destabilizing (e.g. Gonzalez \& Castellanos 1996; Tseluiko et al. 2007). Consequently, despite the results of Kalliadasis \& Homsy (2001), we acknowledge that our solutions may not be stable for sufficiently large values of the electric Weber number. An analysis for electrified flow over topography along the lines of that conducted by Kalliadasis \& Homsy (2001) is left as a topic for future research. Time-dependent calculations of electrified film flow over topography will be presented in future work. 
This research was supported by the EPSRC under grant EP/D052289/1. The work of DTP was supported in part by the National Science Foundation grant DMS-0405639.

\section{REFERENCES}

Abdelouhab, A., Bona, J. L., Felland, M. \& Saut, J.-C. 1989 Nonlocal models for nonlinear dispersive waves. Physica D 40, 360-392.

Alekseenko, S. V., Nakoryakov, V. E. \& Pokusaev, B. G. 1985 Wave formation on a vertical falling liquid film. AIChE J. 31, 1446-1460.

Argyriadi, K., Vlachogiannis, M. \& Bontozoglou, V. 2006 Experimental study of inclined film flow along periodic corrugations: The effect of wall steepness. Phys. Fluids 18, 012102.

Bankoff, S. G., Griffing, E. M. \& Schluter, R. A. 2002 Use of an electric field in an electrostatic liquid film radiator. Ann. N.Y. Acad. Sci. 974, 1-9.

Bankoff, S. G., Miksis, M. J. \& Gwinner, H. Kim R. 1994 Design considerations for the rotating electrostatic liquid-film radiator. Nucl. Engng Des. 149, 441-447.

Benjamin, T. B. 1957 Wave formation in laminar flow down an inclined plane. J. Fluid Mech. 2, 554-574.

Benney, D. J. 1966 Long waves on liquid films. J. Math. Phys. 45, 150-155.

Bertozzi, A. L. \& Brenner, M. P. 1997 Linear stability and transient growth in driven contact lines. Phys. Fluids 9, 530-539.

Bielarz, C. \& Kalliadasis, S. 2003 Time-dependent free-surface thin film flows over topography. Phys. Fluids 15 (9), 2512-2524.

Davis, J. M. \& Troine, S. M. 2005 Generalized linear stability of noninertial coating flows over topographical features. Phys. Fluids 17, 072103.

Decré, M. M. J. \& BARET, J. C. 2003 Gravity-driven flows of low viscosity liquids over twodimensional topographies. J. Fluid Mech. 487, 147-166.

Diez, J. A. \& Kondic, L. 2001 Contact line instabilities of thin liquid films. Phys. Rev. Lett. 86, 632-635.

Fernandez-Parent, C., Lammers, J. H. \& Decré, M. M. J. 1998 Flow of a gravity driven thin liquid film over one-dimensional topographies. Phillips Research Unclassified Report No. UR $823 / 28$.

Gaskell, P. H., Jimack, P. K., Sellier, M. \& Thompson, H. M. 2006 Flow of evaporating, gravity-driven thin liquid films over topography. Phys. Fluids 18, 013601.

Gaskell, P. H., Jimack, P. K., Sellier, M., Thompson, H. M. \& Wilson, M. C. T. 2004 Gravitydriven flow of continuous thin liquid films on non-porous substrates with topography. J. Fluid Mech. 509, 253-280.

Gonzalez, A. \& Castellanos, A. 1996 Nonlinear electrohydrodynamic waves on films falling down an inclined plane. Phys. Rev. E 53, 3573-3578.

Griffing, E. M., Bankoff, S. G., Miksis, M. J. \& Schluter, R. A. 2006 Electrohydrodynamics of thin flowing films. J. Fluids Engng 128.

Gu, F., Liu, C. J., Yuan, X. G. \& Yu, G. C. 2004 CFD simulation of liquid film flow on inclined plates. Chem. Engng Technol. 27, 1099-1104.

Howell, P. D. 2003 Surface-tension-driven flow on a moving curved surface. J. Engng Math. 45, 283-308.

Jensen, O. E., Chini, G. P. \& KInG, J. R. 2004 Thin-film flows near isolated humps and into corners. J. Engng Math. 50, 289-309.

Kalliadasis, S., Bielarz, C. \& Homsy, G. M. 2000 Steady free-surface thin film flows over topography. Phys. Fluids 12, 1889-1898.

Kalliadasis, S. \& Homsy, G. M. 2001 Stability of free-surface thin-film flows over topography. J. Fluid Mech. 448, 387-410.

Kim, H., Bankoff, S. G. \& Miksis, M. J. 1992 The effect of an electrostatic field on film flow down an inclined plane. Phys. Fluids A 4, 2117-2130.

Kim, H., Bankoff, S. G. \& Miksis, M. J. 1994 The cylindrical electrostatic liquid-film radiator for heat rejection in space. Trans. ASME: J. Heat Transfer 116, 986-992. 
Kistler, S. F. \& Schweizer, P. M. 1997 Liquid Film Coating: Scientific Principles and their Technological Implications. Chapman \& Hall.

LIN, S. P. 1974 Finite amplitude sideband stability of a viscous film. J. Fluid Mech. 63, 417-429.

Lister, J. R. 1990 Buoyancy-driven fluid fracture: similarity solutions for the horizontal and vertical propagation of fluid-filled cracks. J. Fluid Mech. 217, 213-239.

LiU, J. \& Gollub, J. P. 1994 Solitary wave dynamics of film flows. Phys. Fluids 6, 1702-1712.

Liu, J., Paul, J. D. \& Gollub, J. P. 1993 Measurements of the primary instabilities of film flows. J. Fluid Mech. 250, 69-101.

Malamataris, N. A. \& Bontozoglou, V. 1999 Computer aided analysis of viscous film flow along an inclined wavy wall. J. Comput. Phys. 154, 372-392.

Malamataris, N. A., Vlachogiannis, M. \& Bontozoglou, V. 2002 Solitary waves on inclined films: Flow structure and binary interactions. Phys. Fluids 14, 1082-1094.

Mazouchi, A. \& Homsy, G. M. 2001 Free surface Stokes flow over topography. Phys. Fluids 13(10), 2751-2761.

Messé, S. \& DeCRÉ, M. M. J. 1997 Experimental study of a gravity driven water film flowing down inclined plates with different patterns. Phillips Research Unclassified Report No. NL-UR 030/97.

Papageorgiou, D. T. \& Petropoulos, P. G. 2004 Generation of interfacial instabilities in charged electrified viscous liquid films. J. Engng Maths 50, 223-240.

Papageorgiou, D. T. \& Vanden-Broeck, J.-M. 2004 Large-amplitude capillary waves in electrified fluid sheets. J. Fluid. Mech. 508, 71-88.

Pozrikidis, C. 1988 The flow of a liquid film along a periodic wall. J. Fluid Mech. 188, 275-300.

Ramaswamy, B., Chippada, S. \& Joo, S. W. 1996 A full-scale numerical study of interfacial instabilities in thin-film flows. J. Fluid Mech. 325, 163-194.

Roy, R. V., Roberts, A. J. \& Simpson, M. E. 2002 A lubrication model of coating flows over a curved substrate in space. J. Fluid Mech. 454, 235-261.

SChwartZ, L. W. \& Weidner, D. E. 1995 Modelling of coating flows on curved surfaces. J. Engng Math. 29, 91-103.

Serifi, K., Malamataris, N. A. \& Bontozoglou, V. 2004 Transient flow and heat transfer phenomena in inclined wavy films. Intl J. Therm. Sci. 43, 761-767.

Shetty, S. \& CERro, R. L. 1993 Flow of a thin film over a periodic surface. Intl J. Multiphase Flow 19, 1013-1027.

Spence, D. A., Sharp, P. W. \& Turcotte, D. L. 1987 Buoyancy-driven crack propagation: a mechanism for magma migration. J. Fluid Mech. 174, 135-153.

Stillwagon, L. E. \& Larson, R. G. 1988 Fundamentals of topographic substrate leveling. J. Appl. Phys. 63, 5251-5258.

Stillwagon, L. E. \& LaRson, R. G. 1990 Leveling of thin films over uneven substrates during spin coating. Phys. Fluids A 2, 1937-1944.

Stocker, R. \& Hosoi, A. E. 2005 Lubrication in a corner. J. Fluid Mech. 544, 353-377.

Tougou, H. 1978 Long waves on a film flow of a viscous fluid down an inclined uneven wall. J. Phys. Soc. Japan 44, 1014-1019.

TRIFOnOv, YU. YA. 1999 Viscous liquid film flows over a periodic surface. Intl J. Multiphase Flow 24, 1139-1161.

Tseluiko, D., Blyth, M. G., Papageorgiou, D. T. \& Vanden-Broeck, J.-M. 2008 Effect of an electric field on the creeping flow of a liquid film down a corrugated wall. (Submitted).

Tseluiko, D. \& Papageorgiou, D. T. 2006 Wave evolution on electrified falling films. J. Fluid Mech. 556, 361-386.

Vlachogiannis, M. \& Bontozoglou, V. 2002 Experiments on laminar film flow along a periodic wall. J. Fluid Mech. 457, 133-156.

WANG, C. Y. 1981 Liquid film flowing slowly down a wavy incline. AIChE $J$ 27, 207-212.

Wang, C. Y. 1984 Thin film flowing down a curved surface. Z. Angew. Math. Phys. 35, 532-544.

Wierschem, A. \& AKsel, N. 2004a Hydraulic jumps and standing waves in gravity-driven flows of viscous liquids in wavy open channels. Phys. Fluids 16, 3868-3877.

Wierschem, A. \& AKsel, N. 2004b Influence of inertia on eddies created in films creeping over strongly undulated substrates. Phys. Fluids 16, 4566-4574. 
Wierschem, A., Scholle, M. \& Aksel, N. 2003 Vortices in film flow over strongly undulated bottom profiles at low Reynolds numbers. Phys. Fluids 15, 426-435.

YıH, C.-H. 1963 Stability of liquid flow down an inclined plane. Phys. Fluids 6, 321-334.

Yoshimura, P. N., Nosoko, T. \& Nagata, T. 1996 Enhancement of mass transfer into a falling laminar liquid film by two-dimensional surface waves: Some experimental observations and modeling. Chem. Engng Sci. 51, 1231-1240.

Zhao, L. \& Cerro, R. L. 1992 Experimental characterization of viscous film flows over complex surfaces. Intl J. Multiphase Flow 18, 495-516. 